CTP-TAMU-11/92

ACT $-1 / 92$

UAHEP922

\title{
The Search for a Realistic Flipped SU(5) String Model
}

\author{
JORGE L. LOPEZ $(a)(b) *$, D. V. NANOPOULOS ${ }^{(a)(b)}$, and KAJIA YUAN ${ }^{(c)}$ \\ (a) Center for Theoretical Physics, Department of Physics, Texas A\&MM University \\ College Station, TX 77843-4242, USA \\ (b) Astroparticle Physics Group, Houston Advanced Research Center (HARC) \\ The Woodlands, TX 7r7381, USA \\ (c) Department of Physics and Astronomy, The University of Alabama \\ Box 870324, Tuscaloosa, AL 35487-0324, USA
}

\begin{abstract}
We present an extensive search for a general class of flipped $S U(5)$ models built within the free fermionic formulation of the heterotic string. We describe a set of algorithms which constitute the basis for a computer program capable of generating systematically the massless spectrum and the superpotential of all possible models within the class we consider. Our search through the huge parameter space to be explored is simplified considerably by the constraint of $N=1$ spacetime supersymmetry and the need for extra $Q, \bar{Q}$ representations beyond the standard ones in order to possibly achieve string gauge coupling unification at scales of $\mathcal{O}\left(10^{18} \mathrm{GeV}\right)$. Our results are remarkably simple and evidence the large degree of redundancy in this kind of constructions. We find one model with gauge group $S U(5) \times U(1)_{\widetilde{Y}} \times S O(10)_{h} \times S U(4)_{h} \times U(1)^{5}$ and fairly acceptable phenomenological properties. We study the $D$ - and $F$-flatness constraints and the symmetry breaking pattern in this model and conclude that string gauge coupling unification is quite possible.
\end{abstract}

February, 1992

* Supported by an ICSC-World Laboratory Scholarship. 


\section{Introduction}

String model-building has provided a new and profuse source of ideas to overcome many of the weaknesses of traditional unified models. Indeed, any string-derived model possesses a definite gauge group whose gauge couplings unify at a calculable high-energy scale, and its matter representations and their gauge and Yukawa interactions are completely determined. In practice this means that one faces a rather constrained problem with little or no room for ways out of potentially phenomenologically disastrous situations. Fortunately (or unfortunately) there is a very large number of models to choose from and one then hopes that an educated path through the morass of possibilities might lead to a model which describes the features of the low-energy world and also predicts new observable phenomena. The purpose of this paper is to pursue one such path through a (hopefully sizeable) portion of this space.

There are several classes of constraints that one can apply to the universe of possibilities to reduce the sample that needs to be considered. The most basic ones are: $N=1$ spacetime supersymmetry and a gauge group below the string scale that either includes the standard model gauge group or embeds it but can be dynamically broken down to it at a lower energy scale. There is also an array of phenomenological constraints that need to be satisfied by the successful model: the low-energy spectrum must contain the three generations of quarks and leptons, and the very accurately measured values of $\sin ^{2} \theta_{w}, \alpha_{e}$, and $\alpha_{3}$ must be reproduced. The fulfillment of both these constraints depends on the details of the path from the Planck scale down to low energies, and are therefore harder to enforce given our present lack of understanding of the mechanics of supersymmetry breaking. At a deeper level of detail we must reproduce the observed values of the quark and lepton masses. Any string-derived model which satisfies all these constraints is a candidate for the correct theory of all particles and interactions, and can be tested by e.g., their predictions for the top-quark, neutrino, higgs, and sparticle masses.

From the string theory point of view there are some organizing principles. It is widely believed that all string models can be thought of as points in a parameter space called the moduli space. Each particular model having fixed values of these parameters, although classes of models with continously connected values of the parameters are very common. The hope is that eventually a string theory principle will be found which will somehow select the energetically most favorable point in this space. In practice, this space has been parceled up into several different 'formulations' of string theory [1] which describe 
(not necessarily non-overlapping) subsets of models. For model-building purposes we have chosen to explore (a portion of) this parameter space with the visor of the so-called free fermionic formulation of the heterotic string [2, 3, 4, 5, 6, 6, 7]. This formulation can be described as a set of model-building rules which is amenable to systematic algebraic and symbolic manipulation and therefore can be translated into a computer code. The description and implementation of such code is one of the objectives of this paper. A further bonus of working within this formulation is the ease with which the terms in the low-energy effective action can be calculated [8], such as the cubic and higher-order superpotential couplings.

String theory also provides a correlation between the gauge group and its allowed matter representations. First of all, these always come in anomaly-free sets (except for a possible pseudo-anomalous $U_{A}(1)$ subgroup). The four-dimensional gauge group is a reflection of the algebra of two-dimensional world-sheet currents called the Kac-Moody algebra [9]. These algebras can be realized at integer values of the 'level' $k=1,2, \ldots$, all of which represent the same gauge group. The allowed matter representations depend on the chosen level which basically determines a cap on the dimensionality of the allowed representations at that level [10,11]. By far the simplest and most common realizations are level one. These allow a very limited set of matter representations which do not include the adjoint representation. This result eliminates from further consideration any of the traditional unified groups (e.g., $\left.S U(5), S O(10), E_{6}\right)$. Higher-level realizations are possible (and they allow the adjoint representation) although in practice these are harder to construct [12, 11]. Besides, the unified groups that would become phenomenologically viable require high levels of the Kac-Moody algebra to accommodate the large representations which appear in traditional model-building with these gauge groups [10]. Limiting ourselves to level-one Kac-Moody algebras we have two classes of models to consider: the class of 'flipped'-like $S U(n)$ models (of which flipped $S U(5)$ [13, 14, 15] is the simplest) and related models [16], or models which contain explicitly the standard model gauge group [17, 18, 19] and therefore require no further symmetry breaking. In this paper we explore the simplest unified models which can be constructed using the simplest Kac-Moody algebras, i.e., the class of flipped $S U(5)$ string models.

There already exists one string-derived realization of a flipped $S U(5)$ model in this formulation, 1 the so-called 'revamped' flipped $S U(5)$ model [20,21,22]. This model has been explored in detail in the literature [24,25,26, 27,28,29] and several of its interesting

1 Flipped $S U(5)$ models have also been constructed in other formulations [23]. 
features have been highlighted, such as the natural apperance of a hierarchical fermion mass spectrum [24,27], adequate low-energy higgs sector [24,27], acceptable proton decay rate [26], the existence of bound states of fractionally charged hidden sector particles called cryptons [25], etc. Despite all these attractive features, gauge coupling unification at a scale of $\mathcal{O}\left(10^{18} \mathrm{GeV}\right)$ as predicted in this model [30,31], is probably not possible [32], or conversely, the low-energy values of $\sin ^{2} \theta_{w}$ and $\alpha_{3}$ are probably not reproducible. In any event, one must consider these statements from the proper perspective since it is tacitly assumed that the matter content of the model does not differ from the minimal one. This is not so in the true string model which possesses several fields beyond the minimal ones at poorly determined intermediate mass scales. Nevertheless, no well motivated choice for these mass scales has improved the situation, and there even exists a no-go theorem to this effect (see below).

Two ways out this impasse have been proposed [33]: string threshold effects on the gauge couplings could reduce the effective unification scale down to $\mathcal{O}\left(10^{16} \mathrm{GeV}\right)$ [34, 35], or new matter fields could delay unification until $\mathcal{O}\left(10^{18} \mathrm{GeV}\right)$ [32, 36]. The first alternative is not viable in models built in the free fermionic formulation since threshold corrections always increase the effective unification scale [31,34]. 目 The minimal field-theoretical extra matter content (beyond the fields in the supersymmetric standard model) needed in the second alternative has been determined to be an extra pair of $Q$ and $D^{c}$ vector-like representations [37, which fit inside a $\mathbf{1 0}$ representation of $S U(5)$. However, in a more complicated situation like for a string-derived flipped $S U(5)$ model, one can only assert that one extra $Q, \bar{Q}$ pair is absolutely necessary. The main purpose of this paper is to search for such models within the chosen formulation.

The organization of this paper is as follows. In Sec. 2 we review the fundamentals of the free fermionic formulation and rephrase its model-building rules to suit our computational purposes. In Sec. 3 we describe our computer algorithms. In Sec. 4 we explore the parameter space for the desired models, and in Sec. 5 we present the results of the extensive computer search. In Secs. 6 and 7 we describe in some detail the most promising model found, and in Sec. 8 we summarize our conclusions.

2 The threshold corrections needed in models where this mechanism may work require rather unnatural values of the moduli fields [34]. 


\section{The free fermionic formulation}

It has become well known that consistent classical string vacua (i.e., "string models") in low spacetime dimensions $\left(d<d_{c}=10\right)$, especially in four dimensions, can be constructed in a variety of formulations [1]. Different formulations correspond to different choices for the two-dimensional world-sheet conformal field theory, so that these extra two-dimensional degrees of freedom, which may or may not admit a spacetime interpretation, together with the two-dimensional conformal fields that describe the observed four-dimensional spacetime, ensure the (super)conformal invariance and modular invariance of the full string theory. In the free fermionic formulation [2, 3, 4, 45,6, ,7], all the internal two-dimensional degrees of freedom are fermionized by utilizing only free world-sheet fermionic fields. The stringy consistency conditions are cast into a set of constraints on the spin-structures [38] of all the world-sheet fermions (i.e., on the boundary conditions of these fermions as they are parallell transported around noncontractible loops on the worldsheet) and a set of constraints on the relative contributions of different spin-structures to the string partition function.

The two-dimensional world-sheet fermions should provide the exact amount of central charge for the Virasoro algebra in order to render the quantum theory free of conformal anomalies, both in the left- and right-moving sectors. For the four-dimensional heterotic string $3, c_{L}=9$ and $c_{R}=22$ are needed; these conditions fix the total number of worldsheet fermions. In the light-cone gauge, in addition to the two left-moving fermionic fields $\psi^{\mu}(\mu=1,2)$ which are the superpartners of the two transverse string coordinates $X^{\mu}$, the internal fermionic content consists of 18 left-moving and 44 right-moving real fermions, respectively. The world-sheet supersymmetry in the left-moving sector is nonlinearly realized among the left-moving fermions which must transform as the adjoint representation of a semi-simple Lie algebra of dimension $3(10-d)=18$ [39]. To achieve $N=1$ spacetime supersymmetry, this algebra must be $S U(2)^{6}$ [7], and therefore we can group the left-moving fermions into 6 triplets $\left(\chi^{\ell}, y^{\ell}, \omega^{\ell}\right)(\ell=1,2, \ldots, 6)$, each transforming as the adjoint representation of $S U(2)$, with the following supercurrent

$$
T_{F}=\psi^{\mu} \partial_{z} X_{\mu}+\sum_{\ell=1}^{6} \chi^{\ell} y^{\ell} \omega^{\ell}
$$

3 We follow the convention that left-movers are supersymmetric and right-movers are nonsupersymmetric. 
where $T_{F}$ must be periodic or antiperiodic on the world-sheet, corresponding to spacetime fermions or spacetime bosons.

In addition to the requirement of (super)conformal invariance, the most important constraints on the spin-structures of world-sheet fermions are obtained by considering modular invariance. The basic idea is to investigate the properties of the string partition and correlation functions under modular transformations, and then search for the solutions that accommodate modular invariance. In the free fermionic formulation, besides the constraints that modular invariance imposes on the one-loop string partition function, further constraints can be obtained in two different approaches: (1) requiring modular invariance and factorization of multiloop partition functions [2,3]; or (2) performing a "physical sensible projection" from the space of world-sheet states onto the subspace of physical states which ensures the correct spin-statistics connection [4]. These two approaches have been shown to be entirely equivalent [5,6]. It turns out that the results of such analysis can be described as a set of model-building rules. We now give a self-contained account of these rules.

The first set of rules restricts the spin-structure assignments of the world-sheet fermions. Before stating these rules, it is helpful to introduce the spin-structure vectors which specify unambiguously the spin-structures of all world-sheet fermions. Consider a $g$-loop world-sheet $\Sigma_{g}$; there are $2 g$ noncontractible loops. For each loop, a spin-structure of a set of world-sheet fermions is given by a orthogonal matrix representation of the first homotopy group $\pi\left(\Sigma_{g}\right)$ which is non-abelian for $g \geq 2$. In the approach of Ref. [4], since only the one-loop partition function is relevant, one can just specify the boundary conditions of the world-sheet fermions around the two noncontractible loops of the torus, and they are mutually commuting. However, in the approach of Refs. [2] 3], on a two- or higher-loop world-sheet, the analysis of multi-loop modular invariance and factorization becomes a rather delicate affair, since non-commuting boundary conditions are allowed [3]. Nevertheless, simplification can be achieved by restricting oneself to the subset of mutually commuting boundary conditions, which amounts to replacing $\pi\left(\Sigma_{g}\right)$ by its abelianized version, i.e., the first homology group $H_{1}\left(\Sigma_{g}\right)$. Under this restriction, even in the approach of Refs. [2,3] ( which we follow closely in this paper) the spin-structure matrices can be simultaneously diagonalized for all noncontractible loops. As a result, for any noncontractible loop $v$, one can simply specify the corresponding spin-structure by a spin-structure vector

$$
v=\left[v\left(f_{1}\right), \ldots, v\left(f_{n}\right)\right], \quad v(f) \in(-1,1]
$$


where $n=n_{r}+n_{c}, n_{r}$ and $n_{c}$ are the numbers of the real and complex fermions. The component $v(f)$ represents the boundary condition of fermion $f$ under parallel-transport around this loop

$$
f \rightarrow-e^{i \pi v(f)} f
$$

It is convenient to introduce a sign for each spin-structure vector $v$ as follows, $\delta_{v}=e^{i \pi v\left(\psi^{\mu}\right)}$. This sign distinguishes between spacetime bosons $\left(\delta_{v}=+1\right)$ and spacetime fermions $\left(\delta_{v}=\right.$ -1) [2,3]. Clearly, one can choose to define the spin-structure vector differently. In fact, the $W$-vector used in Ref. [4] and the $V$-vector used in Ref. [5] are related to our $v$-vector by $v(f)=1-2 W(f)=-2 V(f)$.

It was shown in Ref. [3] that if one only considers the special spin-structure vectors whose components are rational numbers, then all possible rational spin-structure vectors that give rise to consistent string models must form a finite additive group $\Xi$, which can be generated by a basis $\mathcal{B}=\left\{b_{1}, b_{2}, \ldots, b_{p}\right\}$ of a finite number of spin-structure basis vectors. Each basis vector $b_{i}$ has order $N_{b_{i}}$, which is defined as the smallest positive integer such that

$$
N_{b_{i}} b_{i}=\mathbf{0} \quad(\bmod 2)
$$

Here $\mathbf{0}$ is a special spin-structure vector which assigns antiperiodic boundary conditions to all world-sheet fermions; we will refer to it as the Neveu-Schwarz vector in the rest of this paper. The basis $\mathcal{B}$ must obey the following constraints:

(A1) It can be chosen to be a canonical basis, such that

$$
\sum_{i=1}^{p} m_{i} b_{i}=\mathbf{0} \quad(\bmod 2) \Longleftrightarrow m_{i}=0 \quad\left(\bmod N_{b_{i}}\right) \forall i
$$

In terms of this canonical basis, an arbitrary spin-structure vector $v \in \Xi$ is an integer linear combination of basis vectors reduced in interval $(-1,1]$, which we express as

$$
v=\left[\sum_{i=1}^{p} v_{i} b_{i}\right], \quad v_{i}=0,1,2, \ldots,\left(N_{b_{i}}-1\right) .
$$

(A2) One of the basis vectors, which we choose to be the first one $b_{1}$, satisfies

$$
\frac{1}{2} N_{b_{1}} b_{1}=\mathbf{1} \quad(\bmod 2)
$$

Here 1 is another special spin-structure vector which assigns periodic boundary conditions to all world-sheet fermions; we will refer to it as the Ramond vector for convenience. 
Although the general forms of such $b_{1}$ vector have been tabulated [7], we will choose $b_{1}=\mathbf{1}$ for simplicity, hence $N_{\mathbf{1}}=2$.

(A3) If $N_{b_{i} b_{j}}$ is the least common multiple of $N_{b_{i}}$ and $N_{b_{j}}$, then

$$
N_{b_{i} b_{j}}\left(b_{i} \cdot b_{j}\right)=0 \quad(\bmod 4)
$$

(A4) For any $b_{i} \in \mathcal{B}$,

$$
N_{b_{i}}\left(b_{i} \cdot b_{i}\right)=\left\{\begin{array}{lll}
0 & (\bmod 4) & \text { if } N_{b_{i}} \text { is odd } \\
0 & (\bmod 8) & \text { if } N_{b_{i}} \text { is even }
\end{array}\right.
$$

However, if $N_{b_{i}}\left(b_{i}+\mathbf{1}\right)=\mathbf{0}(\bmod 4)$, then $b_{i}$ must satisfy a stronger condition

$$
N_{b_{i}}\left(b_{i} \cdot b_{i}\right)=N_{b_{i}}(\mathbf{1} \cdot \mathbf{1}) \quad(\bmod 16) .
$$

In $(2.8)-(2.10)$, the dot product between two spin-structure vectors $u$ and $v$ is defined by国

$$
u \cdot v=\left\{\frac{1}{2} \sum_{\substack{\text { real } \\ \text { left }}}+\sum_{\substack{\text { complex } \\ \text { left }}}-\frac{1}{2} \sum_{\substack{\text { real } \\ \text { right }}}-\sum_{\substack{\text { complex } \\ \text { right }}}\right\} u(f) v(f) .
$$

(A5) The number of real fermions that are simultaneously periodic in any given three basis vectors $b_{i}, b_{j}, b_{k}$ is even [5], i.e.,

$$
\sum_{\text {real }} b_{i}(f) b_{j}(f) b_{k}(f)=0 \quad(\bmod 2) \quad(1 \leq i, j, k \leq p) .
$$

(A6) The spin-structure corresponding to each $b_{i}$ should be an automorphism of the Lie algebra defining the world-sheet supercurrent, and all such automorphisms must commute with one another. In the case of our interest, the world-sheet Lie algebra is $S U(2)^{6}$, and the possible commuting sets of automorphisms have been tabulated in Ref. [7]. This rule restricts in a rather intricate way the possible sets of basis vectors which are allowed to coexist. We defer to the next section a further discussion of this constraint.

Having reviewed the constraints of the spin-structure vectors, we now move on to the second set of rules which restricts the relative contributions of different spin-structures to the one-loop string partition function. As it was shown in Refs. [3, 2], this set of rules can be given in terms of some proper normalized coefficients which enter the one-loop partition

4 This differs from the definition in Refs. [4,5] by a overall sign due to the different conventions used. 
function and depend upon the spin-structures. Since such one-loop spin-structure coefficients are pure phases [3], any direct computer implementation would involve complex numbers. In order to avoid this unnecessary complication, we use a $k$-matrix notation similar to that introduced in Refs. [四, 5]. More precisely, for a given basis $\mathcal{B}=\left\{b_{1}, b_{2}, \ldots, b_{p}\right\}$, we define a $p \times p$ positive integer matrix (which we call the $k$-matrix) in terms of the oneloop spin-structure coefficients $C\left[\begin{array}{c}b_{i} \\ b_{j}\end{array}\right]$ associated with basis vectors $b_{i}$ and $b_{j}$ (see Ref. [3]), such that its element $k_{i j}$ is given by

$$
C\left[\begin{array}{l}
b_{i} \\
b_{j}
\end{array}\right]=\delta_{b_{i}} e^{2 \pi i k_{i j} / N_{b_{j}}}, \quad\left(1 \leq k_{i j} \leq N_{b_{j}}\right) .
$$

With the $k$-matrix, the second set of model-building rules can be written as follows: (B1) There is freedom in choosing $k_{11}$. In fact, we have $1 \leq k_{11} \leq N_{b_{1}}$ and

$$
8 k_{11}=N_{b_{1}}\left(b_{1} \cdot b_{1}\right) \quad\left(\bmod 4 N_{b_{1}}\right)
$$

or

$$
8 k_{11}=N_{b_{1}}\left[b_{1} \cdot b_{1}-4 b_{1}\left(\psi^{\mu}\right)\right] \quad\left(\bmod 8 N_{b_{1}}\right) .
$$

Since we take $b_{1}=\mathbf{1}$, Eq. (2.14) gives $k_{11}=1$ and Eq. (2.15) gives $k_{11}=2$.

(B2) Besides $k_{11}$, all other diagonal elements $k_{i i}(i \geq 2)$ of the $k$-matrix are completely fixed by the corresponding $k_{i 1}$,

$$
8 k_{i i}=N_{b_{i}}\left[4 k_{i 1}+b_{i} \cdot b_{i}+\mathbf{1} \cdot \mathbf{1}-2\left(2-N_{b_{1}}\right) b_{i}\left(\psi^{\mu}\right)\right] \quad\left(\bmod 8 N_{b_{i}}\right)(i \geq 2) .
$$

Note that the last term on the right side vanishes when $N_{b_{1}}=N_{\mathbf{1}}=2$.

(B3) The elements below the diagonal $k_{i j}(i>j)$ and the corresponding elements above the diagonal are related as follows (for $i \neq j$ ),

$$
4\left(N_{b_{i}} k_{i j}+N_{b_{j}} k_{j i}\right)+2 N_{b_{i}} N_{b_{j}}\left[b_{i}\left(\psi^{\mu}\right)+b_{j}\left(\psi^{\mu}\right)\right]-N_{b_{i}} N_{b_{j}}\left(b_{i} \cdot b_{j}\right)=0 \quad\left(\bmod 4 N_{b_{i}} N_{b_{j}}\right) .
$$

It is easy to see that a $k$-matrix is completely determined by $k_{11}$ and the elements below the diagonal (or equivalently the elements above the diagonal). Therefore, for a given basis $\mathcal{B}$, there are $2 \prod_{i \neq j} g_{b_{i} b_{j}}$ distinct $k$-matrices associated with it [3], where $g_{b_{i} b_{j}}$ $(i \neq j)$ is the greatest common divisor of $N_{b_{i}}$ and $N_{b_{j}}$ (which gives the number of choices for $\left.k_{i j}\right)$.

\footnotetext{
5 Note: Despite its same name, our $k$-matrix definition differs from that in Refs. [4, 5 ].
} 
Given a pair $(\mathcal{B}, k)$, subject to rules $(\mathrm{A} 1)-(\mathrm{A} 6)$ and rules (B1)-(B3) respectively, one can construct a consistent four-dimensional heterotic string model. The total Hilbert space of the model is a direct sum of the sub-Hilbert spaces of all the sectors in the model, each of which corresponds to a spin-structure vector $v$ of form (2.6) and is refered to as the $v$-sector and sometimes denoted as $\mathcal{H}_{v}$. Furthermore, the physical states in the $v$-sector are determined by the generalized GSO-projections [40], which take various forms [3, 团. Strictly speaking, in order to perform the GSO-projections correctly, special care must be taken when dealing with real fermions, because the zero modes of real fermions with periodic boundary conditions contribute to the string partition function [5]. Of course, if all the left- and right-moving real world-sheet fermions in the model can be separately complexified (i.e., described by left- and right-moving complex fermions obtained by pairing up two real fermions that have the same spin-structure) then the model is just a special case of a complex fermion model, and there is no real subtlety involved.

In this paper, however, we would like to consider a more general class of models, in which only $2 n_{L}\left(2 n_{L}<20\right)$ of the left- and $2 n_{R}\left(2 n_{R}<44\right)$ of the right-moving real fermions are complexifiable in the usual sense. Clearly, the two left-moving fermionic fields $\psi^{\mu}$ should always be treated as one complex fermion. (Our choice of $S$-vector (described below) allows complexification of the $\chi^{\ell}$ into three complex fermions $[8$.) In addition, any remaining left- and right-moving real fermions appear in equal numbers, and can be grouped in left-right pairs which share the same boundary conditions (periodic or antiperiodic) for all basis vectors, effectively representing a non-chiral conformal field theory, e.g., an Ising model [8]. This class of pseudo-complex fermion models is a subset of the general class of real fermion models considered in Ref. [5], and is precisely the class of "real fermion models" discussed in Ref. [8]. Restricting ourselves to the pseudo-complex fermion models, we can divide the world-sheet fermions into three categories: (1) $n_{L}$ left-moving complex fermions; (2) $n_{R}$ right-moving complex fermions; (3) $n_{I}$ Ising models each formed from a "residual" left- and a "residual" right-moving real fermion. The numbers of these three types of world-sheet fermions are related by

$$
n_{I}=44-2 n_{R}=20-2 n_{L} .
$$

Accordingly, we can rearrange any spin-structure vector $v$ into three parts: a $n_{L^{-}}$ dimensional vector $v^{L}$, a $n_{R^{-}}$dimensional vector $v^{R}$ and a $n_{I}$-dimensional vector $v^{I}$. For the particular class of basis vectors we will consider in this paper, below we will see that rule 
(A6) implies that the elements in $v^{L}$ and $v^{I}$ can only be either 1 or 0 , whereas the entries in $v^{R}$ can be rational numbers, which describe genuine right-moving complex world-sheet fermions. For any complex world-sheet fermion $f$, one can define the fermionic charge in the $v$-sector as [4,21]

$$
Q_{v}(f)=F_{v}(f)+\frac{1}{2} v(f)
$$

where $F_{v}(f)$ is the fermion number and $v(f)$ the boundary condition. Therefore, for left- and right-moving complex fermions, there are the fermionic charge vectors $Q_{v}^{L}\left(n_{L^{-}}\right.$ dimensional) and $Q_{v}^{R}\left(n_{R}\right.$-dimensional $)$ respectively. For the real fermions in left-right pairs, the concept of the fermionic charge is not applicable. However, in the models under consideration, their effect can be taken into account simply by introducing two operators for each Ising model $f^{I}$ : (1) a "fermion number difference" operator $\Delta F_{v}\left(f^{I}\right)$, which equals the fermion number of the left-moving real fermion minus that of the corresponding rightmoving fermion when $v\left(f^{I}\right)=0$, but equals zero when $v\left(f^{I}\right)=1$; $(2)$ a "chirality" operator $\Gamma_{v}\left(f^{I}\right)$ such that $\Gamma_{v}\left(f^{I}\right)=1$ if $v\left(f^{I}\right)=0$, and $\Gamma_{v}\left(f^{I}\right)= \pm 1$ if $v\left(f^{I}\right)=1$. Collectively, we have two well-defined $n_{I}$-dimensional vectors $\Delta F_{v}^{I}$ and $\Gamma_{v}^{I}$.

In terms of $Q^{L}, Q^{R}, \Delta F^{I}$ and $\Gamma^{I}$, we can rewrite the GSO-projections in a very convenient way. In fact, for all basis vectors $b_{i}(1 \leq i \leq p)$, the physical states in the $v$-sector must satisfy

$$
b_{i}^{L} \cdot Q_{v}^{L}-b_{i}^{R} \cdot Q_{v}^{R}+b_{i}^{I} \cdot \Delta F_{v}^{I}+\frac{1}{2} b_{i}^{I} \cdot\left(\Gamma_{v}^{I}-\mathbf{1}^{I}\right)=v\left(\psi^{\mu}\right)+b_{i}\left(\psi^{\mu}\right)+2 \sum_{j=1}^{p} \frac{k_{i j} v_{j}}{N_{b_{j}}} \quad(\bmod 2)
$$

where $\mathbf{1}^{I}$ is the $n_{I^{-}}$-dimensional vector with all the entries equal 1 . When a particular string state of $v$-sector that satisfies (2.20) is obtained, its quantum numbers (with which the corresponding vertex operator can be constructed) are completely determined by the three vectors $Q_{v}^{L}, Q_{v}^{R}$ and $\Gamma_{v}^{I}$ [ $\left[\mathbb{8}\right.$. More explicitly, the first entry of $Q_{v}^{L}$ describes the spacetime spin of the state, the next three entries give the charges under the three $U(1)$ 's which make up the conserved $U_{J}(1)$ current of the $N=2$ world-sheet supersymmetry algebra, and the remaining entries $\left(n_{L}-4\right)$ are the charges under residual left-moving global $U(1)$ symmetries. The right-moving fermion charge vector $Q_{v}^{R}$ encodes partially 6 the charges

6 It is quite often the case that a full matter representation of the gauge group is formed by states that come from more than one sector of the model. 
under the Kac-Moody currents and therefore the gauge quantum numbers. In particular, the rank of the total gauge group $G$ equals the dimension of $Q_{v}^{R}$, that is

$$
\operatorname{rank}(\mathrm{G})=n_{R} .
$$

Finally, the vector $\Gamma_{v}^{I}$ is related to the eigenvalues of the order (disorder) operator for all

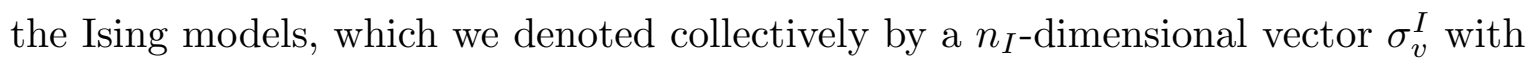

$$
\sigma_{v}\left(f^{I}\right)=v\left(f^{I}\right) \Gamma_{v}\left(f^{I}\right) .
$$

The mass of a string state in the $v$-sector is given by the following formula [3]

$$
\begin{aligned}
M^{2} & =-\frac{1}{2}+\frac{1}{8}\left\{\frac{1}{2} \sum_{\substack{\text { real } \\
\text { left }}}+\sum_{\substack{\text { complex } \\
\text { left }}}\right\} v(f) v(f)+\sum_{\text {left }} \text { (frequencies), } \\
& =-1+\frac{1}{8}\left\{\frac{1}{2} \sum_{\substack{\text { real } \\
\text { right }}}+\sum_{\substack{\text { complex } \\
\text { right }}}\right\} v(f) v(f)+\sum_{\text {right }} \text { (frequencies). }
\end{aligned}
$$

Where the oscillator frequencies for a fermion $f$ are

$$
\frac{1+v(f)}{2}+\text { integer, }
$$

and if $f$ is complex, its complex conjugate $f^{*}$ has frequencies

$$
\frac{1-v(f)}{2}+\text { integer. }
$$

Also, the bosonic oscillators contribute integer frequencies.

\section{Description of the computer program}

One of the advantages of the free fermionic formulation is that the rules for modelbuilding are quite simple and can be computerized, which makes a fairly systematical study of the string models built in this formulation possible. We are aware of one such attempt in the literature [41], which was based on the formulation of Ref. [4]. However, in Ref. 41], only complex fermion models were considered, while the more interesting models with real fermions were not taken into account. In order to investigate the pseudo-complex fermion models, we have independently developed a computer program, based on the formalism described in Sec. 2 and with an approach different from that of Ref. [41]. Our computer software is written in FORTRAN. The bulk of the program is devoted to the generation of the massless spectrum of a pseudo-complex fermion model once the basis and the consistent $k$-matrix of the model are given. In addition, our program is also able to calculate the complete trilinear superpotential and identify all the fermion Yukawa couplings of the model. In this section we explain our method and discuss some algorithms involved. 


\subsection{The basis and the $k$-matrix}

To obtain a basis $\mathcal{B}$ that obeys the rules (A1)-(A6), we should first decide on the first basis vector $b_{1}$. An obvious choice that we make throughout this paper is $b_{1}=\mathbf{1}$. As long as $b_{1}$ is fixed, rule (A2) is not relevant, and the remaining task is to generate additional basis vectors such that the other rules are satisfied. We choose the second basis vector $b_{2}$ to be the "supersymmetry generator" $S$, which allows the existence of spacetime supersymmetry by giving rise to massless gravitinos. Of the several possible forms of $S$ that are compatible with the choice $b_{1}=\mathbf{1}$ [7], we choose the simplest one, which is the only one that has been used for model-building in the literature, namely

$$
S=\left(1100100100100100100: \mathbf{0}_{R}\right)
$$

Here the first entry is reserved for the two transverse $\psi^{\mu}$ treated as a single complex fermion; the following 18 entries correspond to the left-moving internal fermions treated here as 6 triplets $\left(\chi^{\ell}, y^{\ell}, \omega^{\ell}\right)$; and the vector $\mathbf{0}_{R}$ after the colon, which separates the left-movers from the right-movers, simply indicates that all right-moving fermions have antiperiodic boundary conditions.

An immediate bonus of choice (3.1) is that the possible forms for the left-moving part of all other basis vectors can be rather easily determined. In fact, if $S$ is given as in (3.1), then in order for the basis vectors to satisfy rule (A6), the left-moving internal fermions must have either periodic or antiperiodic boundary conditions [7], organized as 6 triplets $\left(\chi^{\ell}, y^{\ell}, \omega^{\ell}\right)$ of real fermions. Furthermore, in any basis vector $b_{i}$, the boundary conditions for these fermions must satisfy [7]

$$
\begin{aligned}
& b_{i}\left(\psi^{\mu}\right)=b_{i}\left(\chi^{\ell}\right)+b_{i}\left(y^{\ell}\right)+b_{i}\left(\omega^{\ell}\right) \quad(\bmod 2), \\
& b_{i}\left(\psi^{\mu}\right), b_{i}\left(\chi^{\ell}\right), b_{i}\left(y^{\ell}\right), b_{i}\left(\omega^{\ell}\right) \in\{0,1\} \quad(1 \leq \ell \leq 6) .
\end{aligned}
$$

Because of our choice of $S$, the otherwise rather intricate check of rule (A6) can be done very easily in the class of models we consider. In addition, the specific form of the vector $S$ also gives us some useful information on the right-moving part of all other basis vectors. From (3.1) it is easy to see that only $y^{\ell}$ and $\omega^{\ell}(\ell=1, \ldots, 6)$ are available to serve as the "residual" left-moving real fermions (since the $\chi^{\ell}$ can always be complexified). In order to obtain the pseudo-complex fermion models, we need to allow equal numbers of left- and right-moving real fermions. Therefore, we deduce that the first 12 entries of the right-moving part (corresponding to 12 real fermions denoted by $\bar{y}^{\ell}$ and $\bar{\omega}^{\ell}$ ) of all basis 
vectors will be either 0 or 1 . Furthermore, we can always treat the remaining right-moving fermions as complex, i.e., the last 16 entries of each basis vector can be rational numbers in the interval $(-1,1]$.

Any test vector with the above general features has a chance of being a viable basis vector. Once such a vector is generated (either by hand or by computer) our program first checks whether or not it satisfies rules (A3) and (A4), by calculating the dot product of this vector with all the other basis vectors that have been previously entered into the basis. If the test vector fails this step, the program discards it and repeats the same procedure for another test vector, until a good candidate is found. At this point, the program records the relevant information about this new basis vector, such as its order and the associated dot products. This whole process can be repeated as many times as one wishes and is controled by an interactive command. After having obtained a set of several basis vectors, this set would constitute a basis $\mathcal{B}$ if rule (A1) and (A5) are also satisfied. The algorithm for checking rule (A1) is very simple. Our program first generates all possible linear combinations of form (2.6) with the set of basis vectors, and then looks for the Neveu-Schwarz vector $\mathbf{0}$, which should appear only once, when all the "coordinates" $v_{i}=0$, if the basis is canonical. As far as rule (A5) is concerned, it can simply be checked using (2.12). However, since we are particularly interested in the pseudo-complex fermion models, we have designated a special subprogram to check whether the model is complexifiable as described in Sec. 2. Since all complexifiable models satisfy rule (A5), once this is established we would have obtained an allowed basis $\mathcal{B}$ which would give rise to a pseudo-complex fermion model. It is worthwhile to point out that in our approach we start with basis vectors of the general form described above, and do not specify $n_{L}, n_{R}$ or $n_{I}$. These numbers are determined by the program after the complexification procedure is successful. The advantage of this approach is that our program can treat equally any pseudo-complex fermion model with gauge group of rank between 16 and 22 (see Eqs. (2.18) and (2.21)).

Having generated a basis $\mathcal{B}$, all consistent $k$-matrices can be systematically obtained by using (2.13)-(2.17). In practice, the number of possibilities is prohibitively large and one has to resort to random generation of these matrices (see Sec. 5). However, even in the random approach there are still many distinct $k$-matrices, although they do not necessarily lead to distinct or phenomenologically acceptable string models. To avoid a large cosmological constant at the string scale, we are interested only in models with spacetime supersymmetry. Furthemore, to obtain chiral fermions this better be $N=$ 
1 supersymmetry (as opposed to $N=2$ or 4 which are also possible). Such models can be obtained if the basis $\mathcal{B}$ contains enough basis vectors to break the initial $N=4$ supersymmetry down to $N=1$ [7]. However, since the GSO-projections relevant to the massless gravitinos in the model (we need one and only one gravitino for $N=1$ ) depend on the choice of $k$-matrix, only a subset of the $k$-matrices will give $N=1$ supersymmetric models in the end. We have written a special subprogram which calculates the massless gravitinos provided by the $S$-sector immediately after a $k$-matrix is generated. In this way, the program acts on only those selected $k$-matrices and discards all others. In Sec. 4.2 we will show that given a specific basis, the constraints on the $k$-matrices imposed by requiring $N=1$ supersymmetry can be worked out explicitly, in which case we just use the explicit constraints to test for $N=1$ supersymmetry.

\subsection{The massless sectors}

To find the massless spectrum of a string model, we only need to consider those sectors which could possibly contain massless states. All we need are the basis $\mathcal{B}$ and the mass formula (2.23). For an arbitrary $v$-sector, let us consider the following two quantities

$$
\begin{aligned}
& M_{L}=\left\{\frac{1}{2} \sum_{\substack{\text { real } \\
\text { left }}}+\sum_{\substack{\text { complex } \\
\text { left }}}\right\} v(f) v(f)=v^{L} \cdot v^{L}+\frac{1}{2} v^{I} \cdot v^{I}, \\
& M_{R}=\left\{\frac{1}{2} \sum_{\substack{\text { real } \\
\text { right }}}+\sum_{\substack{\text { complex } \\
\text { right }}}\right\} v(f) v(f)=v^{R} \cdot v^{R}+\frac{1}{2} v^{I} \cdot v^{I} .
\end{aligned}
$$

In the models under consideration, since the left-moving part of all basis vectors is made up of 0's and 1's, the left-moving part of any spin-structure vector $v$ of form (2.6) will only have 0 or 1 entries as well. Therefore, there are only two possible forms of massless states in the left-moving part: the non-degenerate bosonic vacuum with a single fermionic oscillator

of frequency $\frac{1}{2}$ acting on it, and the degenerate fermionic vacuum with no oscillator at all. Accordingly, a $v$-sector will provide massless states only if $M_{L}=0$ or $M_{L}=4$.

For the right-moving part, since the last 16 entries can be arbitrary rational numbers in the interval $(-1,1]$, a similar analysis is to certain extent basis-dependent. Nevertheless, if we confine ourselves to models in which the only rational numbers that appear in the basis vectors are $\pm \frac{1}{2}$, then these would also be the only rational numbers in any vector $v$. As a result, the possible right-moving fermionic frequencies are $\frac{1}{4}, \frac{1}{2}$ and $\frac{3}{4}$, and in order for a $v$-sector of such models to provide massless states, it has to have $M_{R}=0,2,4,6,8$. 


\subsection{The gauge group}

The massless vector states of a heterotic string model, which are created by exciting the left-moving antiperiodic transverse fermions $\psi^{\mu}$ and the right-moving internal fermions, are the gauge bosons. 0 These come from massless sectors with $M_{L}=0$ and transform as the adjoint representation of the gauge group $G$ of the string model. Since the right-moving complex fermions are the building blocks of the two-dimensional Kac-Moody currents that underlie the four-dimensional gauge symmetry, the gauge group $G$ of each pseudocomplex fermion model can be systematically and unambiguously determined by studying the fermionic charge vectors $Q_{v}^{R}$ for all the sectors with $M_{L}=0$. First of all (as mentioned in Sec. 2), the number of right-moving complex fermions $n_{R}$, which only depends on the basis $\mathcal{B}$, gives the rank of $G$. Secondly but most importantly, the $Q_{v}^{R}$ vectors which label the gauge bosons always constitute the roots of the Lie algebra associated with the gauge group $G[4]$.

We start with the gauge bosons in the Neveu-Schwarz sector (0-sector) which is always present in all models (see (2.6)). Due to the existence of the "residual" antiperiodic rightmoving real fermions (not present in complex fermion models), to get gauge bosons we can either excite two right-moving complex fermions or excite one right-moving complex fermion and one such "residual" right-moving real fermion (in both cases we have $M_{R}=0$, i.e., '00' gauge bosons). In the first case we get right-moving fermionic charge vectors of the form

$$
Q_{0}^{R}\left(f_{i}\right)= \pm \delta_{i j} \pm \delta_{i k} \quad(j \neq k)
$$

as well as some vanishing ones. In the second case, these vectors take the form

$$
Q_{0}^{R}\left(f_{i}\right)= \pm \delta_{i j}
$$

The Neveu-Schwarz sector is special in that the $Q_{\mathbf{0}}^{R}$ vectors of the gauge bosons in this sector themselves constitute the roots of a Lie algebra associated with a subgroup of $G$ with of same rank, which we refer to as the "minimal" gauge group $G_{m}$ of the string model. Clearly, for the simple models in which the Neveu-Schwarz sector is the only sector giving gauge bosons, $G_{m}$ and $G$ coincide. It is remarkable to see that, from (3.4) and

7 Gauge bosons which arise by exciting left-moving internal degrees of freedom preclude the existence of chiral (massless) spacetime fermions if the resulting gauge group is abelian (nonabelian) 42]. 
(3.5), not only the $Q_{0}^{R}$ vectors correspond to the roots of $G_{m}$, but their elements also specify precisely the coordinates of these roots in the standard orthonormal basis [43]. In addition, the forms of (3.4) and (3.5) imply that the minimal gauge group $G_{m}$ in general is a direct product of simple factors of $S O(2 m), S U(m+1)$ and $S O(2 m+1)$, as well as some $U(1)$ factors. Here, the non-simply-laced groups $S O(2 m+1)$ arise simply because the "residual" right-moving real fermions are allowed in pseudo-complex fermion models (see (3.5)). Since roots always appear in pairs of opposite sign, we only need to consider the positive roots, i.e., the $Q_{0}^{R}$ whose first nonvanishing entry is positive. In the Neveu-Schwarz sector, our program first constructs the 462 positive roots of the Lie algebra $D_{22}$, which corresponds to the primordial gauge symmetry $S O(44)$ of four-dimensional heterotic string models built in the free fermionic formulation. The positive roots of the minimal gauge group $G_{m}$ are then obtained by performing the GSO-projections (2.20) which in this case reduce to

$$
-b_{i}^{R} \cdot Q_{\mathbf{0}}^{R}+b_{i}^{I} \cdot \Delta F_{\mathbf{0}}^{I}=0 \quad(\bmod 2) .
$$

Because the $Q_{0}^{R}$ vectors are $n_{R}$-dimensional, and in the pseudo-complex fermion models we have $16 \leq n_{R} \leq 22$, only the first $n_{R}$ entries of the 462 positive roots of $D_{22}$ enter in (3.6). (When $n_{R}<22$, the first $n_{R}$ entries of some of these positive roots are all zeros; such $n_{R}$-dimensional zero vectors are obviously not positive, and our program discards them automatically.) After the positive roots in the Neveu-Schwarz sector are found this way, the program is instructed by an interactive command, to either find the simple roots from these positive roots (according to the algorithm which we will describe below) and therefore pin down the minimal gauge group $G_{m}$ for some testing purposes, or to go on searching for all the additional gauge bosons in other sectors and finally find the total gauge group $G$ of the model.

Besides the Neveu-Schwarz sector, any $v$-sectors of the model with $M_{L}=0$ could also provide gauge bosons. The most common gauge bosons of this type have $M_{R}=8$ (i.e., '08' gauge bosons); in practice we have also found models with '06' gauge bosons. The procedure to find these additional gauge bosons is the same: we start from a set of appropriate fermionic charge vectors $Q_{v}^{R}$ in each of such sectors, keeping only those vectors whose first nonvanishing entry is positive, and then find the subset which survives the GSO-projections. In contrast with the Neveu-Schwarz sector, two differences are worth mentioning. First, for the Neveu-Schwarz sector the GSO-projections (3.6) are independent of the $k$-matrix. So once a basis $\mathcal{B}$ is given, the minimal gauge group $G_{m}$ is completely 
determined (prior to the generation of the $k$-matrices). For other sectors that give gauge bosons, the corresponding GSO-projections become

$$
-b_{i}^{R} \cdot Q_{v}^{R}+b_{i}^{I} \cdot \Delta F_{v}^{I}=2 \sum_{j=1}^{p} \frac{k_{i j} v_{j}}{N_{b_{j}}} \quad(\bmod 2),
$$

which clearly depend on the $k$-matrix. As a result, the total gauge group $G$ of the string model varies with different choices for the $k$-matrix. Second, although the gauge bosons of the Neveu-Schwarz sector give rise to the minimal gauge group $G_{m}$, the gauge bosons from other sectors by themselves do not have this property. Instead, the additional gauge bosons transform as certain representations of the minimal group $G_{m}$, such that all these representations of $G_{m}$ combine with the adjoint representation coming from the NeveuSchwarz sector to make up precisely the adjoint representation of an enlarged group $G$.

Having found all the positive roots, to uniquely identify the gauge group $G$, the next step is to find the simple roots and calculate the Cartan matrix. The same idea was followed in Ref. [41], where a recursive procedure was used to find the simple roots according to the property of their inner products with each other. Here we take a simpler approach, which is based on the following definition of the simple roots: A simple root is a positive root that cannot be written as the sum of two other positive roots [43]. Our program first adds the positive roots together pairwise, then it compares all the positive roots with these two-root pairs and finds the simple roots. This way we end up with $n_{\text {srt }} \leq n_{R}$ simple roots in some arbitrary order.

To determine explicitly the factors in the gauge group we proceed in two steps. We first isolate a set of the simple roots which forms a subspace by itself, i.e., that would give a block diagonal submatrix in the Cartan matrix in a suitable basis. That is, $\alpha_{i} \cdot \alpha_{j} \neq 0$ for $i, j \in \mathbf{b}$, but $\alpha_{i} \cdot \alpha_{k}=0$ for $i \in \mathbf{b}, k \notin \mathbf{b}$. The number of roots in this block is the rank $r_{i}$ of the sought after gauge group factor $G_{i}$. We then calculate the Cartan matrix $C_{i j}$ of this block of simple roots. If $r_{i}=1$ and $C_{11}=0(2)$ then $G_{i}=U(1)(S U(2))$. For $r_{i}>1$ the determination of $G_{i}$ can be made unambigously from $r_{i}$ and $\operatorname{det} C$, as seen from Table I, and the following additional considerations: for simplicity we identify $C_{l}$ with $B_{l}$, although proper identification is possible; we distinguish $B_{7}$ from $E_{7}$ by the fact that the Cartan matrix of the former is not symmetric; and we take into account explictly the isomorphism $A_{3} \approx D_{3}$. The above procedure is repeated until all simple roots are exhausted and then a symbolic expression for the total gauge group is printed out. In order to be able to identify the matter representations automatically, it is necessary to reorder the simple roots in each 
block in a standard way (such as the one given in Ref. 450). This is possible once the $G_{i}$ have been identified. However, the algorithm is non-trivial and cumbersome and will not be described here.

When $n_{\text {srt }}<n_{R}$, the gauge group $G$ also contains $\left(n_{R}-n_{\text {srt }}\right) U(1)$ factors. In the models that we are considering, the $U(1)$ gauge group can arise in two ways: (1) If a single right-moving complex fermion has unique boundary conditions so that no other right-moving complex fermions have the same ones in all the basis vectors, then this single complex fermion provides a $U(1)$ factor, and the corresponding $U(1)$ charge of a given representation is specified by the value of its fermionic charge in that sector; (2) If a unitary group arises through the embedding $S U(m) \times U(1) \subset S O(2 m)$, then a $U(1)$ appears as a "trace", and the $U(1)$ charge of a given representation in this case is the sum of the fermionic charges over all fermions in the trace. However, there are more complicated embeddings for $U(1)$ factors, identification of which needs to be done on a non-automated case-by-case basis.

\subsection{The matter states}

The massless matter states can come from massless sectors with $M_{L}=0$ or 4 . Similar to the case of massless gauge bosons, the gauge group properties of the massless matter states are carried by their right-moving fermionic charge vectors $Q_{v}^{R}$, which are the weights of the corresponding representations of the Lie algebra associated with the gauge group $G$ [4. (As explained in Sec. 2, the left-moving fermionic charge vectors $Q_{v}^{L}$ also contain useful quantum numbers of the states [8].)

Since we only consider models with $N=1$ spacetime supersymmetry, the matter states form $N=1$ supermultiplets. A full $N=1$ supermultiplet is provided by states from a $v$-sector and the corresponding $(v+S)$-sector, so we always treat them together. The fermionic and scalar components of an $N=1$ supermultiplet transform under the same representation of the gauge group, but their left-moving quantum numbers are different. For the fermionic components we choose to keep states with positive spacetime helicity, i.e., $Q_{v}^{L}\left(\psi^{\mu}\right)=\frac{1}{2}$. The correct left-moving fermionic charges for the corresponding scalar components then satisfy 8

$$
\sum_{i=2,3,4} Q_{v}^{L}\left(f_{i}\right) Q_{g r a v}^{L}\left(f_{i}\right)=\frac{1}{2}
$$

8 This result is a generalization of Eq. (37) in Ref. 8] [with the following notational relations: $Q_{v}^{L}\left(f_{i}\right) \leftrightarrow \alpha, \beta, \gamma ; Q_{g r a v}^{L}\left(f_{i}\right) \leftrightarrow \alpha_{12}, \alpha_{34}, \alpha_{56}$ ] for the case of an arbitrary component of the gravitino vertex operator. 
where $Q_{g r a v}^{L}$ is the fermionic charge vector of the (positive-helicity) gravitino in the model, which our program has determined, and the sum runs over the three complexified leftmoving fermions constructed from the $\chi^{\ell}$.

The way in which we find the right-moving fermionic charge vectors $Q_{v}^{R}$ for all massless matter states is essentially the same. We first construct a set of appropriate fermionic charges vectors $Q_{v}^{R}$ for each sector, and only keep those that survive the GSO-projections (2.20). Since the weights of Lie algebra representations do not necessarily come in pairs, we have to consider both positive and negative weights. To specify the irreducible representations of the matter states, we only need to keep track of the highest weights that appear in the end. Again, differently from the approach of Ref. 441, we find the highest weights by first converting all weights into the corresponding Dynkin labels using the properly reordered simple roots, and then keeping only those weights whose Dynkin labels are all non-negative integers [45].

The identification of the transformation properties of the selected set of weights under the various $G_{i}$ groups is done via a look-up table. That is, the program knows the Dynkin labels of all allowed representations under all gauge groups which can occur in a level-one Kac-Moody construction (a limited set [10]). Looping over all $G_{i}$ we obtain a symbolic label for each state, e.g., 10-1-1 for a 10 under $S U(5)$ and a singlet under other two $G_{i}$ 's. For later purposes, the multiplicity and conjugacy classes 45,10] (see Sec. 3.5) of each representation are also calculated and saved. We also determine the contribution to the anomaly of each $S U(N), N \geq 3$ subgroup $G_{i}$ by each of the states in the spectrum, as follows

$$
A_{i}=\sum_{R_{j}} a_{i}\left(R_{j}\right) \operatorname{dim}\left(R_{j} / G_{i}\right),
$$

where $a(R)=(N-3) !(N-2 n) /(N-n-1) !(n-1)$ ! for totally antisymmetric representations (the only ones that can occur at level one [10]) of $\operatorname{dimension} \operatorname{dim}(R)=\left(\begin{array}{l}N \\ n\end{array}\right)$ 46]. In Eq. (3.9), $\operatorname{dim}\left(R_{j} / G_{i}\right)$ is the dimension of $R_{j}$ under the subgroups other than $G_{i}$. Since all these anomalies must vanish, this constitutes a rather valuable check of the correctness of the program.

\subsection{The superpotential}

Once the full massless spectrum is available, the program computes the cubic superpotential couplings using the techniques of Ref. [8]. This is done by forming all possible $\phi_{1} \phi_{2} \phi_{3}$ sets and determining whether such coupling is invariant under the various 
two-dimensional world-sheet symmetries. One of these is equivalent to four-dimensional gauge invariance. This is checked using the conjugacy classes determined earlier: un$\operatorname{der} G_{i}$ we must have $c_{i}\left(\phi_{1}\right)+c_{i}\left(\phi_{2}\right)+c_{i}\left(\phi_{3}\right)=0 \bmod \left(d_{i}\right)$. For example, for $S U(n)$, $c(\mathbf{1})=0, c(\mathbf{n})=1, c(\mathbf{n}(\mathbf{n}-\mathbf{1}) / \mathbf{2})=2, c(\overline{\mathbf{R}})=-c(\mathbf{R})$, and $d=n$; for $S O(2 n)$, $c(\mathbf{1})=0, c(\mathbf{n})=2, c\left(\mathbf{2}^{\mathbf{n}-\mathbf{1}}\right)=-1$, and $d=4$. We also compute explicitly the conformal field theory correlators for the various Ising models (corresponding to each left-right real fermion pair). For cubic couplings these can be tabulated explicitly using the following multiplication table

$$
\begin{aligned}
& \begin{array}{lllll}
I & \sigma_{+} & \sigma_{-} & f
\end{array} \\
& \begin{array}{l}
I \\
\sigma_{+} \\
\sigma_{-} \\
f
\end{array}\left(\begin{array}{cccc}
I & \sigma_{+} & \sigma_{-} & f \\
\sigma_{+} & I & f & \sigma_{-} \\
\sigma_{-} & f & I & \sigma_{+} \\
f & \sigma_{-} & \sigma_{+} & I
\end{array}\right),
\end{aligned}
$$

where $\sigma_{ \pm}$are the eigenvalues of the chirality operator defined in Eq. (2.22) (also known as the order/disorder Ising model operators), and $f$ represents the corresponding left- or right-moving real fermion. For example, the correlator $\left\langle\sigma_{+} \sigma_{-} f\right\rangle$ is nonvanishing because $\sigma_{+} \sigma_{-} f \rightarrow f f \rightarrow I$, according to Eq. (3.10).

For flipped $S U(5)$ models, among the various cubic couplings there are some of the form: $10_{a} \cdot \overline{5} \cdot \overline{5}, 10_{b} \cdot 10_{c} \cdot 5_{d}, \overline{5} \cdot 1 \cdot 5_{e}$. These are candidates for the up- and down-quark type and lepton-type fermion Yukawa couplings. The code identifies these couplings and determines whether it is possible to have non-vanishing top, bottom, and tau Yukawa couplings at the cubic level, i.e., whether there are couplings such that $10_{a}=10_{b}$ or $10_{a}=10_{c}$, and $5_{d}=5_{e}$.

A separate portion of the code determines the set of possibly non-vanishing quartic and higher-order superpotential couplings according to the (significantly more complicated) rules of Ref. [8]. All we want to point out here is that the multiplication table given above can be used to determine whether a high-point Ising model correlator (corresponding to real fermion degrees of freedom not involved in the picture changing operations) vanishes. This test reduces considerably the number of terms that need to scanned by hand. In fact, at the quartic level this program gives only non-vanishing couplings. 


\section{The space of flipped SU(5) models}

The computer program described in the previous section can be used to study generic models built within the free fermionic formulation. As discussed in the Introduction, we restrict ourselves here to models with the flipped $S U(5)$ group as the observable part of the total gauge group. This constraint is rather weak once one realizes the size of the space to be explored. Instead of doing an exhaustive search of all possible bases and $k$ matrices, we choose a well motivated subset of the space of basis vectors (seven vectors) and consider a general class of vectors ' $\alpha$ ' which effect the breaking $S O(10) \rightarrow S U(5) \times U(1)$, as discussed below. We also consider all the possible $k$-matrices compatible with the bases under consideration.

\subsection{The choice of basis}

We study a basis of eight vectors $\mathcal{B}=\left\{\mathbf{1}, S, b_{1}, b_{2}, b_{3}, b_{4}, b_{5}, \alpha\right\}$, the first seven of which are given in Table II. The choice of $\mathbf{1}$ and $S$ was discussed in Secs. 2 and 3.1. The presence of vectors $b_{1}$ and $b_{2}$ is necessary to reduce the spacetime supersymmetry from $N=4$ down to $N=1$. The precise form of these vectors is basically fixed by self-consistency constraints [7,47]. The presence of $b_{3}$ is necessary to enlarge the massless spectrum and possibly accommodate three generations of chiral fermions. It has even been argued [47] that the $b_{1}, b_{2}, b_{3}$ set is a unique one which must be present in any three-generation model. Basis vectors $b_{4}$ and $b_{5}$ are needed to allow for symmetry-breaking Higgs representations in the massless spectrum. Their precise forms have not been fully classified, although their left-moving entries are constrained by self-consistency conditions (see Sec. 2). Also, these vectors should not destroy or enhance the spacetime supersymmetry of the model. In sum, given the form of $S$, the first five vectors in $\mathcal{B}$ are to a large extent "written in stone", whereas the forms of $b_{4}$ and $b_{5}$ have been chosen as in the revamped model [20].

The vector $\alpha$ serves the purpose of breaking the resulting $S O(10)$ gauge symmetry down to $S U(5) \times U(1)$, and must therefore contain rational entries which we take for simplicity to be $\frac{1}{2}$. We study the following generic form of the $\alpha$-vector

$$
\alpha=\left(00 y^{1} y^{1} \cdots 0 y^{6} y^{6}: \bar{y}^{1} \bar{y}^{2} \bar{y}^{3} \bar{y}^{4} \bar{y}^{5} \bar{y}^{6} \bar{w}^{1} \bar{w}^{2} \bar{w}^{3} \bar{w}^{4} \bar{w}^{5} \bar{w}^{6} \frac{1}{2} \frac{1}{2} \frac{1}{2} \frac{1}{2} \frac{1}{2} \frac{1}{2} \frac{1}{2} \frac{1}{2} \frac{1}{2} \frac{1}{2} \frac{1}{2} \frac{1}{2} 1100\right)
$$

where Eq. (3.2) has been used to set $\alpha\left(w^{\ell}\right)=\alpha\left(y^{\ell}\right)$. The choice for the fixed entries was made to a large extent to follow the revamped example. With the choice of $b_{1,2,3,4,5}$,

9 The other possible forms of $S$ [7] have remained virtually unexplored in the literature. 
the complexification procedure indicates that (depending on $\alpha$ ) the possible gauge groups will have rank 16-19. The actual rank in excess of 16 depends on how many of the three $U(1)$ symmetries left unbroken by $b_{1,2,3,4,5}$ are also left unbroken by $\alpha$. These symmetries are generated by the following complexified pairs of real fermions, $U_{a}: \bar{w}^{2} \bar{w}^{3}, U_{b}: \bar{y}^{1} \bar{w}^{6}$, and $U_{c}: \bar{y}^{4} \bar{y}^{5}$. It is convenient to divide up the possible forms of $\alpha$ into eight classes, according to the number and type of $U_{a, b, c}$ symmetries that they break or do not break. This classification is given in Table III. (The revamped model belongs to class 3a.)

\subsection{The constraints on the $k$-matrix}

Given the basis $\mathcal{B}$ with eight vectors, the corresponding $k$-matrix is $8 \times 8$, but only the 28 lower-diagonal entries are independent (see Sec. 2). As shown below, this leads to a very large number of possible $k$-matrices, most of which may not necessarily lead to phenomenologically interesting models. We know present three classes of constraints on the $k$-matrix which guarantee $N=1$ spacetime supersymmetric models, and allow the possibility of three generations of matter fields and symmetry-breaking Higgs fields.

The GSO projections in Eqs. 2.20) can be simplified considerably for potential gravitino states in the $S$-sector, as follows

$$
b_{i}^{L} \cdot Q_{S}^{L}=1+b_{i}\left(\psi^{\mu}\right)+k_{i 2}(\bmod 2) .
$$

For $b_{i}=\alpha$ we have $\alpha^{L} \cdot Q_{S}^{L}=0\left(\right.$ since $\alpha\left(\chi^{\ell}\right)=0$ ) and $\alpha\left(\psi^{\mu}\right)=0$, therefore $k_{82}=1$. This constraint follows solely from the assumed form of $\alpha$ in Eq. (4.1). Analogously one can show that $b_{1}^{L} \cdot Q_{S}^{L}=b_{4}^{L} \cdot Q_{S}^{L}$ and $b_{2}^{L} \cdot Q_{S}^{L}=b_{5}^{L} \cdot Q_{S}^{L}$, and therefore $k_{62}=k_{32}$ and $k_{72}=k_{42}$ if we want to preserve at least one gravitino. It can also be shown that $k_{52}$ is determined as follows $k_{52}=1+k_{21}+k_{32}+k_{42}(\bmod 2)$. If we pick the positive-helicity gravitino state, i.e., $Q_{S}^{L}\left(\psi^{\mu}\right)=\frac{1}{2}$, then the eight possibilities for $Q_{S}^{L}\left(\chi^{1,2}, \chi^{3,4}, \chi^{5,6}\right)$ follow uniquely from the values of $k_{32,42,52}$ as follows: $Q_{S}^{L}\left(\chi^{12,34,56}\right)=-\frac{1}{2}(-1)^{k_{32,42,52}}$.

We must also have representations left from the $b_{1,2,3}$-sectors. In this case a special property of these vectors, namely $b_{i} \cdot b_{j}=b_{i}\left(\psi^{\mu}\right)-\sum_{k=1}^{5} b_{i}\left(\bar{\psi}^{k}\right)=-4$ (where $\bar{\psi}^{\mu}$ are the first five right-moving complex fermions) allows one to deduce that

$$
b_{2}^{L} \cdot Q_{b_{1}}^{L}-b_{2}^{R} \cdot Q_{b_{1}}^{R}=b_{3}^{L} \cdot Q_{b_{1}}^{L}-b_{3}^{R} \cdot Q_{b_{1}}^{R}=Q_{b_{1}}^{L}\left(\psi^{\mu}\right)-\sum_{k=1}^{5} Q_{b_{1}}^{R}\left(\bar{\psi}^{k}\right) .
$$

It can then be shown that we must have $k_{43}=k_{53}$ for the states in the $b_{1}$-sector to remain. A similar exercise for the $b_{2,3}$-sectors yields the constraint $k_{43}=k_{53}=k_{54}$. Further study 
of the effect of the remaining GSO projections indicates that if $k_{43}=k_{53}=k_{54}=1(2)$, then we will get $\overline{\mathbf{1 6}}, \overline{\mathbf{1 6}},\left(\mathbf{1 6}, \mathbf{1 6}^{\prime}\right)$ representations from each of $b_{1,2,3}$.

An analogous argument applied to the $b_{4,5}$-sectors yields further constraints on the $k$-matrix, as follows

$$
\begin{array}{ll}
k_{61}=k_{63} \Rightarrow k_{64}=k_{65}, & k_{61} \neq k_{63} \Rightarrow k_{64} \neq k_{65}, \\
k_{71}=k_{74} \Rightarrow k_{73}=k_{75}, & k_{71} \neq k_{74} \Rightarrow k_{73} \neq k_{75} .
\end{array}
$$

\subsection{The possible models}

Given our choice of basis $\mathcal{B}=\left\{1, S, b_{1}, b_{2}, b_{3}, b_{4}, b_{5}, \alpha\right\}$, it is convenient to determine the possible sets of $S U(5)$ representations coming from the sectors $\mathcal{H}_{b_{1}, b_{2}, b_{3}, b_{4}, b_{5}}$ since these will contribute the Ramond states of the observable spectrum. It is not hard to show that the possible representations are as follows:

$$
\begin{aligned}
& b_{1}, b_{2}, b_{3}: \overline{\mathbf{5}}+\mathbf{1 0} \text { or } 2 \cdot \mathbf{1 0} \text { or } 2 \cdot \overline{\mathbf{5}} \\
& b_{4}, b_{5}: \mathbf{5}+\mathbf{1 0} \text { or } \overline{\mathbf{5}}+\overline{\mathbf{1 0}} \text { or } \mathbf{1 0}+\overline{\mathbf{1 0}} \text { or } \mathbf{5}+\overline{\mathbf{5}} \\
& b_{4}+b_{5}:(\mathbf{5}+\overline{\mathbf{5}})_{v} \text { or } 2 \cdot \mathbf{5} \text { or } 2 \cdot \overline{\mathbf{5}}
\end{aligned}
$$

where the $\mathbf{5}, \overline{\mathbf{5}}, \mathbf{1 0}, \overline{\mathbf{1 0}}$ representations are pieces of $\mathbf{1 6}, \overline{\mathbf{1 6}}$ representations of $S O(10)$, whereas $(\mathbf{5}+\overline{\mathbf{5}})_{v}=\mathbf{1 0}$ of $S O(10)$. We can now form all possible combinations of the above representations such that the total $S U(5)$ anomaly is zero, 10 and $n_{10} \geq 4, n_{\overline{10}} \geq 1$. Five possibilities arise: $n_{10} / n_{\overline{10}}: 4 / 1,4 / 2,5 / 1,5 / 2,6 / 2$. (These go with $n_{5} / n_{\overline{5}}: 2 / 5,2 / 4,1 / 5,1 / 4,0 / 4$.) Of these possibilities, only $4 / 1$ and $5 / 2$ models are likely to yield three light 10's (or equivalently $n_{g}=n_{10}-n_{\overline{10}}=3$ ). The important point here is that the $4 / 1$ and $5 / 2$ models are obtained only when the $(\mathbf{5}+\overline{\mathbf{5}})_{v}$ representations are the ones contributed by $\mathcal{H}_{b 4+b 5}$. This requirement imposes a constraint on the possible $\alpha$-vectors as follows.

Since the $S O(10)$ symmetry is not broken down to $S U(5) \times U(1)$ until the $\alpha$-vector GSO projection is performed, whether we get $(\mathbf{5}+\overline{\mathbf{5}})_{v}$ or $2 \cdot \mathbf{5}$ or $2 \cdot \overline{\mathbf{5}}$ from the $b_{4}+b_{5^{-}}$ sector depends only on this last GSO projection. Reverting to the old notation for the GSO projection, the coefficient which matters is $C\left[\begin{array}{c}b_{4}+b_{5} \\ \alpha\end{array}\right]= \pm 1( \pm i)$ for $\left(b_{4}+b_{5}\right) \cdot \alpha=$ even (odd). By explicit calculation one can show that if $C\left[\begin{array}{c}b_{4}+b_{5} \\ \alpha\end{array}\right]= \pm i$, then $(\mathbf{5}+\overline{\mathbf{5}})_{v}$ will survive if $\alpha\left(y^{2}\right)=\alpha\left(y^{3}\right)\left(y^{2} \bar{y}^{2}\right.$ and $y^{3} \bar{y}^{3}$ form two Ising models), otherwise we get $2 \cdot \mathbf{5}$ or $2 \cdot \overline{\mathbf{5}}$. Given

10 Otherwise a non-trivial mixture with the hidden sector must occur. (States from the NSsector contribute zero to the anomaly.) 
the form of $\alpha$ in Eq. (4.1), it follows that $\alpha\left(w^{2}\right)=\alpha\left(w^{3}\right)$ and therefore $w^{2} w^{3}$ can be complexified and the $U_{a}: \bar{w}^{2} \bar{w}^{3}$ symmetry remains unbroken. Analogously, one can show that if $C\left[\begin{array}{c}b_{4}+b_{5} \\ \alpha\end{array}\right]= \pm 1$, then $(\mathbf{5}+\overline{\mathbf{5}})_{v}$ survives if $\alpha\left(y^{2}\right) \neq \alpha\left(y^{3}\right)$ and therefore $\alpha\left(w^{2}\right) \neq \alpha\left(w^{3}\right)$ and $U_{a}$ is broken. In sum, if $\left(b_{4}+b_{5}\right) \cdot \alpha=$ even (odd) then $U_{a}$ will be broken (unbroken). Now,

$\left(b_{4}+b_{5}\right) \cdot \alpha=-1+\frac{1}{2}\left\{\alpha\left(y^{2}\right)-\alpha\left(\bar{y}^{2}\right)+\alpha\left(y^{3}\right)-\alpha\left(\bar{y}^{3}\right)+\alpha\left(w^{1}\right)-\alpha\left(\bar{w}^{1}\right)+\alpha\left(w^{4}\right)-\alpha\left(\bar{w}^{4}\right)\right\}=-1$,

since $y^{2} \bar{y}^{2}, y^{3} \bar{y}^{3}, w^{1} \bar{w}^{1}, w^{4} \bar{w}^{4}$ always form Ising models. Therefore $U_{a}$ must remain unbroken and from Table III we see that only $\alpha$-vectors from classes 1,2a,2b, and 3a will respect this. The universe of possibilities then consists of $\alpha$-vectors from these classes and the set of distinct $k$-matrices compatible with each basis. Furthermore, only $5 / 2$ models will contain the extra pair of $Q, \bar{Q}$ representations required for gauge coupling unification.

\section{The computerized search}

Given the large number of possibilities, it is clear that one will benefit from a statistical approach to model generation. However, even random generation of a statistically significant number of $k$-matrices (for a fixed $\alpha$ ) is prohibitive: a basis of $p$ vectors goes with an $p \times p k$-matrix, whose $p(p-1) / 2$ lower diagonal entries are independent. In our case $p=8$ and there are $2^{28} \approx 268$ million possible $k$-matrices. Fortunately the constraints on the $k$-matrix derived in Sec. 4.2 are very powerful. Extensive random generation of $k$-matrices indicates that there are further redundancy factors to be obtained, as follows:

(i) $N=1$ supersymmetry determines $k_{52,62,72,82}$ once $k_{21,32,42}$ are given. However, the eight choices for $k_{21,32,42}$ (which correspond to the eight possible choices for the supersymmetry generator) give equivalent models. We then get a $2^{7}$ reduction factor.

(ii) To obtain states from $\mathcal{H}_{b_{1}, b_{2}, b_{3}}$ we need to impose $k_{43}=k_{53}=k_{54}=1,2$. If the model ends up being a $5 / 2$ (or $2 / 5$ ) model, the specific choice just picks $5 / 2$ over $2 / 5$ (i.e., interchanges $\mathbf{1 0} \leftrightarrow \overline{\mathbf{1 0}})$. This gives a $2^{3}$ reduction factor.

(iii) There is no obvious analytic constraint on the entries $k_{31,41,51}$, and heuristically we find that their values do not affect the phenomenological properties of the models. 11 We therefore fix them; a $2^{3}$ reduction factor.

11 They may even give rise to equivalent models, as expected from the internal discrete symmetries associated with the various Ising models. 
(iv) Finally, we have the analytical correlations among $k_{61,63,64,65}$ and $k_{71,73,74,75}$ to obtain states from $\mathcal{H}_{b 4, b 5}$. A reduction factor of $2^{2}$.

The combined analytical and heuristical reduction factor is $2^{15}$ which reduces the universe of possible $k$-matrices down to $2^{13}=8192$. This number of $k$-matrices is in practice still quite large and makes random generation of $k$-matrices belonging to this class practically necessary to be able to explore several $\alpha$-vectors. A word of caution: since the number of possibilities is finite, one must make sure that each generated $k$-matrix is distinct from all previous ones.12 This is not hard to do; we simply identify each $k$ matrix by the decimal equivalent of the 13-digit binary number formed by its independent entries in a fixed order. In order to gain confidence on statistical results (e.g., percentages of models with a given feature) obtained by sampling $\lesssim 10 \%$ of the possible models, we generated all $8192 k$-matrices for one $\alpha$-vector and found the exact results to be in complete agreement with those obtained statistically from smaller samples.13

The $\alpha$-vectors which we consider introduce a large dimension in the space of possible models. Each of these $\alpha$-vectors has 24 free real fermion entries ( $2^{24}$ possibilities), although these are highly constrained. Rule (A6) (see Eq. (3.2)) requires $\alpha\left(y^{\ell}\right)=\alpha\left(w^{\ell}\right)$ for this type of $\alpha$-vectors (they all have $\alpha\left(\chi^{\ell}\right)=0$ ). There are also six more constraints on the 24 entries, one from rule (A4) and five from rule (A3) (the constraint from $b_{i}=\mathbf{1}$ follows from rule (A4), and that from $b_{i}=S$ is automatically satisfied for our choice of $\alpha$-vector). Finally, the splitting of $\alpha$-vectors into classes (see Sec. 4.1) introduces correlations among three pairs of entries. Therefore, there are $24-6-1-5-3=9$ independent real entries in any given $\alpha$-vector, a total of $2^{9}=512$ possibilities.

The program described in Sec. 3 is capable of producing a large amount of output. It was therefore necessary to screen possible models, i.e., calculate the whole spectrum and cubic Yukawa couplings, and keep only summary information about each model (e.g., $k$-matrix number, gauge group, $n_{10} / n_{\overline{10}}$, fermion Yukawa couplings, number and type of hidden sector fields, etc.). With the $k$-matrix information it was then easy to retrieve any particular model afterwards.

12 This is not very important for small samples of $k$-matrices since these obey the following probability distribution $n_{\text {distinct }}=N\left(1-e^{-n_{\text {trials }} / N}\right) \approx n_{\text {trials }}+\mathcal{O}\left(n_{\text {trials }}^{2} / N\right)(N=8192$ in this case).

13 This run took $\approx 40$ hours of cputime in a Silicon Graphics 340 S computer. In comparison, the $2^{28} k$-matrices would take $\approx 153$ years of cputime! 
The results of our search with the general class of $\alpha$-vectors we consider can be summarized as follows:

(a) Class 3a. There are two rank-17 gauge groups possible for any $\alpha$-vector in this class (the $k$-matrix determines which one occurs): $S U(5) \times S U(4) \times S U(2)^{4} \times U(1)^{6}$ and $S U(5) \times S U(4) \times S O(10) \times U(1)^{5}$. We did not find any $5 / 2$ model with the $\alpha$-vectors explored and for all possible choices of $k$-matrices. The revamped model (a $4 / 1$ model) is a prototype of models belonging to this class.

(b) Classes $2 a$ and $2 b$. There are two rank-18 gauge groups possible for each $\alpha$-vector: $S U(5) \times S U(4) \times S U(2)^{4} \times U(1)^{7}$ and $S U(5) \times S U(4) \times S O(10) \times U(1)^{6}$. All $5 / 2$ models found (these occur $1 / 8$ of the time; about 1000 per $\alpha$-vector choice) come with the second gauge group. They also have the same number of massless fields (67), and the same number and type of hidden matter fields ( $\operatorname{six} 4, \overline{4}$ and seven $\mathbf{6}$ of $S U(4)$, and three 10 of $S O(10))$. These models fall into two subgroups: half of them have a 2/3/2 Yukawa set (2 up-quark-type, 3 down-quark-type, and 2 lepton-type cubic Yukawa couplings) and the other half have a 1/3/2 Yukawa set.

(c) Class 1. There is a large number of rank-19 gauge groups possible in this case, although they do not occur for all $\alpha$-vectors (some are more 'prolific' than others). The following is a list of the 13 gauge groups which we have been able to identify:

$$
\begin{aligned}
& S U(5) \times S U(4) \times\left\{\begin{array}{l}
S U(2)^{4} \times U(1)^{8} \\
S U(3)^{2} \times S U(2)^{2} \times U(1)^{6} \\
S U(3) \times S U(2)^{3} \times U(1)^{7} \\
S O(10) \times U(1)^{7}
\end{array}\right. \\
& S U(5) \times S U(5) \times\left\{\begin{array}{l}
S O(10) \times U(1)^{6} \\
E_{6} \times U(1)^{5}
\end{array}\right. \\
& S U(5) \times S U(6) \times\left\{\begin{array}{l}
S U(2)^{3} \times U(1)^{7} \\
S U(3)^{2} \times S U(2) \times U(1)^{5}
\end{array}\right. \\
& S U(7) \times S U(4) \times\left\{\begin{array}{l}
S U(2)^{3} \times U(1)^{7} \\
S U(3) \times S U(2)^{2} \times U(1)^{6}
\end{array}\right. \\
& S U(7) \times S U(6) \times\left\{\begin{array}{l}
S U(2)^{2} \times U(1)^{6} \\
S U(3) \times S U(2) \times U(1)^{5}
\end{array}\right. \\
& S U(9) \times S O(10) \times U(1)^{6}
\end{aligned}
$$

It is interesting to note that (contrary to naive expectations) the addition of the $\alpha$ vector to effect the breaking down to flipped $S U(5)$, can quite likely enlarge the final gauge group and leave no such group after all. There are two kinds of $\alpha$-vectors in this class which give $5 / 2$ models: 
(c1) The 'price'-like $\alpha$-vectors, one of which was used in Ref. [32] to construct the first $5 / 2$ flipped $S U(5)$ model in the literature. In this case 5/2 models (which occur $1 / 4$ of the time) always come with the gauge group $S U(5) \times S U(5) \times S O(10) \times$ $U(1)^{6}$ and do not possess right-handed leptons in their massless spectra (they also have problems with the anomalous $U_{A}(1)$ cancellation), and are therefore irrelevant for phenomenological purposes 14

(c2) The other type of $\alpha$-vectors give $5 / 2$ models ( $1 / 8$ of the time) with gauge group $S U(5) \times S U(4) \times S O(10) \times U(1)^{7}$ and have no problems with right-handed leptons or cancellation of $U_{A}(1)$. These models have the same hidden matter spectrum as those $5 / 2$ models belonging to classes $2 \mathrm{a}$ and $2 \mathrm{~b}$, and they also split into two subgroups: half of them have $1 / 3 / 2$ and the other half have $1 / 4 / 2$ Yukawa sets.15

\section{The phenomenology of the various models}

In the previous section we exhibited the list of possible $5 / 2$ models found under our assumptions about the basis vectors. Given the Yukawa couplings of the various models one can easily convince oneself that only the models that belong to classes $2 \mathrm{a}$ or $2 \mathrm{~b}$ which have the $2 / 3 / 2$ Yukawa set are of any interest. Indeed, in models with only 1 up-quark-type Yukawa coupling (i.e., $1 / 3 / 2$ or $1 / 4 / 2)$ one must identify that coupling $\left(F_{t} \bar{f} \bar{h}\right)$ with the one for the top quark, since no other such coupling appears at cubic, quartic, or quintic order in the superpotential. The problem is that $F_{t}$ does not appear in terms of the form $F_{t} F h$ at cubic, quartic, or quintic order, and therefore we do not expect a (sizeable) bottom Yukawa coupling. We thus concentrate on the $2 / 3 / 2$ models (in comparison, the fairly successful revamped model is a $2 / 3 / 3$ (although $4 / 1$ ) model).

\footnotetext{
14 In Ref. [32 the gauge group for the model presented was incorrectly determined to be $S U(5) \times S U(4) \times S O(10) \times U(1)^{7}$, and so were some of its massless representations. This problem may have arisen because contributions of additional gauge bosons belonging to new '08' sectors may have been overlooked.

15 There is a further subdivision inside each of these sets into two different kinds of $1 / 3 / 2$ and $1 / 4 / 2$ Yukawa sets.
} 


\subsection{The model}

The model we consider in some detail here belongs to class $2 \mathrm{~b}$ (although all $2 / 3 / 2$ models in classes $2 \mathrm{a}$ and $2 \mathrm{~b}$ are equivalent to this one) and has the following $\alpha$-vector

$$
\alpha=\left(00000000000000000011: 000001011001 \frac{1}{2} \frac{1}{2} \frac{1}{2} \frac{1}{2} \frac{1}{2} \frac{1}{2} \frac{1}{2} \frac{1}{2} \frac{1}{2} \frac{1}{2} \frac{1}{2} \frac{1}{2} 1100\right),
$$

and $k$-matrix (this is the same one as the one for the revamped model, although there are 512 such $k$-matrices that will give the same results)

$$
k=\left(\begin{array}{llllllll}
2 & 1 & 2 & 2 & 2 & 2 & 2 & 1 \\
1 & 1 & 1 & 1 & 1 & 1 & 1 & 4 \\
2 & 2 & 2 & 2 & 2 & 2 & 2 & 1 \\
2 & 2 & 2 & 2 & 2 & 2 & 2 & 1 \\
2 & 2 & 2 & 2 & 2 & 2 & 2 & 4 \\
2 & 2 & 2 & 2 & 2 & 2 & 2 & 1 \\
2 & 2 & 2 & 2 & 2 & 2 & 2 & 3 \\
2 & 1 & 1 & 1 & 1 & 1 & 2 & 3
\end{array}\right) .
$$

We start by listing in Table IV the massless fields and their transformation properties under the rank 18 gauge group $S U(5) \times U(1)_{\widetilde{Y}} \times S O(10)_{h} \times S U(4)_{h} \times U(1)^{5}$. The cubic and quartic superpotential terms are easily calculated [8]. We obtain

$$
\begin{aligned}
W_{3}=g \sqrt{2}\{ & F_{0} F_{1} h_{1}+F_{2} F_{2} h_{2}+F_{4} F_{4} h_{1}+F_{4} \bar{f}_{5} \bar{h}_{45}+F_{3} \bar{f}_{3} \bar{h}_{3}+\bar{f}_{2} l_{2}^{c} h_{2}+\bar{f}_{5} l_{5}^{c} h_{2} \\
& +\frac{1}{\sqrt{2}} F_{4} \bar{F}_{5} \phi_{3}+\frac{1}{2} F_{4} \bar{F}_{4} \Phi_{0}+\bar{F}_{4} \bar{F}_{4} \bar{h}_{1}+\bar{F}_{5} \bar{F}_{5} \bar{h}_{2} \\
& +\left(h_{1} \bar{h}_{2} \Phi_{12}+h_{2} \bar{h}_{3} \Phi_{23}+h_{3} \bar{h}_{1} \Phi_{31}+h_{3} \bar{h}_{45} \bar{\phi}_{45}+\text { h.c. }\right) \\
& +\frac{1}{2}\left(\phi_{45} \bar{\phi}_{45}+\phi^{+} \bar{\phi}^{+}+\phi^{-} \bar{\phi}^{-}+\phi_{i} \bar{\phi}_{i}+h_{45} \bar{h}_{45}\right) \Phi_{3}+\left(\eta_{1} \bar{\eta}_{2}+\bar{\eta}_{1} \eta_{2}\right) \Phi_{0} \\
& +\left(\phi_{3} \bar{\phi}_{4}+\bar{\phi}_{3} \phi_{4}\right) \Phi_{5}+\left(\Phi_{12} \Phi_{23} \Phi_{31}+\Phi_{12} \phi^{+} \phi^{-}+\Phi_{12} \phi_{i} \phi_{i}+\text { h.c. }\right) \\
& +T_{1} T_{1} \Phi_{31}+T_{3} T_{3} \Phi_{31} \\
& +D_{6} D_{6} \Phi_{23}+D_{1} D_{2} \bar{\Phi}_{23}+D_{5} D_{5} \bar{\Phi}_{23}+D_{7} D_{7} \bar{\Phi}_{31}+D_{3} D_{3} \Phi_{31} \\
& +\frac{1}{2} D_{5} D_{6} \Phi_{0}+\frac{1}{\sqrt{2}} D_{5} D_{7} \bar{\phi}_{3} \\
& +\widetilde{F}_{4} \widetilde{\bar{F}}_{6} \bar{\Phi}_{12}+\frac{1}{2} F_{3} \widetilde{\bar{F}}_{4} \Phi_{0}+\frac{1}{2} F_{2} \widetilde{\bar{F}}_{5} \Phi_{3}+\widetilde{F}_{6} \widetilde{\bar{F}}_{4} \phi^{+}+\frac{1}{\sqrt{2}} \widetilde{F}_{5} \widetilde{\bar{F}}_{4} \phi_{4} \\
& \left.+\widetilde{F}_{1} \widetilde{\bar{F}}_{2} D_{5}+\widetilde{F}_{2} \widetilde{\bar{F}}_{4} l_{2}^{c}\right\}
\end{aligned}
$$

and

$$
\begin{aligned}
W_{4}= & F_{2} \bar{f}_{2} \overline{\bar{h}}_{45} \bar{\phi}_{4}+F_{3} \bar{F}_{4} D_{4} D_{6}+F_{3} \bar{F}_{5} D_{4} D_{7} \\
& +l_{3}^{c} \widetilde{\bar{F}}_{3} \widetilde{\bar{F}}_{6} D_{7}+l_{5}^{c} \widetilde{F}_{2} \widetilde{\bar{F}}_{3} \bar{\phi}_{3}+\widetilde{F}_{1} \widetilde{\bar{F}}_{3}\left(\phi^{+} \bar{\phi}_{3}+\bar{\phi}^{-} \phi_{3}\right) \\
& +\widetilde{\bar{F}}_{3} \widetilde{\bar{F}}_{5} D_{7} \bar{\phi}^{-}+\widetilde{F}_{2} \widetilde{F}_{5} D_{3} \phi^{-}+\widetilde{F}_{2} \widetilde{F}_{6} D_{3} \phi_{4}+\widetilde{F}_{5} \widetilde{\bar{F}}_{1} D_{2} D_{7} \\
& +\widetilde{F}_{5} \widetilde{\bar{F}}_{2} D_{1} D_{7}+\widetilde{F}_{3} \widetilde{\bar{F}}_{3} D_{3} D_{6}+\widetilde{F}_{4} \widetilde{\bar{F}}_{3} D_{4} D_{7}+\widetilde{F}_{5} \widetilde{\bar{F}}_{4} D_{5} D_{7} .
\end{aligned}
$$

Calculable coefficients [8] have been omitted from $W_{4}$. 


\subsection{F- and D-flatness}

To preserve unbroken supergravity at the string scale, the D-terms of all $U(1)$ symmetries and all the F-terms must vanish, i.e.,

$$
\begin{aligned}
\langle W\rangle & =\left\langle\frac{\partial W}{\partial \phi_{i}}\right\rangle=0, \\
\left\langle D_{A}\right\rangle & =\sum_{i} q_{A}^{i}\left|\left\langle\phi_{i}\right\rangle\right|^{2}+\epsilon=0, \\
\left\langle D_{a}\right\rangle & =\sum_{i} q_{a}^{i}\left|\left\langle\phi_{i}\right\rangle\right|^{2}=0, \quad a=1,2,3,4, \\
\left\langle D_{\widetilde{Y}}\right\rangle & =\sum_{i} \widetilde{Y}_{i}\left|\left\langle\phi_{i}\right\rangle\right|^{2}=0,
\end{aligned}
$$

where $\epsilon=g^{2} \operatorname{Tr} U_{A} / 192 \pi^{2}$. In these formulas, $q_{A}^{i}$ is the charge of the $i$-th field under the anomalous $U_{A}(1)$ [48]: $U_{A}=\left(1 / \operatorname{Tr} U_{A}\right) \sum_{i=1}^{5}\left[\operatorname{Tr} U_{i}\right] U_{i}, q_{a}^{i}$ are the charges under the $5-1=4$ orthogonal traceless linear combinations, and $\widetilde{Y}_{i}$ are the charges under (the anomaly-free) $U(1)_{\widetilde{Y}}$. We obtain

$$
U_{A}=\left(-36 U_{1}-12 U_{2}+24 U_{3}-12 U_{5}\right) / \operatorname{Tr} U_{A}, \quad \operatorname{Tr} U_{A}=46.4758,
$$

and therefore16 $\epsilon=\left(3.7 \times g \times 10^{17} \mathrm{GeV}\right)^{2}$. Note that $\operatorname{Tr} U_{4}=0$; in fact, almost all singlets (except $\eta_{1,2}, \bar{\eta}_{1,2}$ ) are neutral under this $U(1)$ symmetry. The rotated traceless $U(1)$ 's (excluding $U_{4}$ ) can be written as follows (proper normalization is understood, although not relevant in what follows)

$$
\begin{aligned}
& U_{A}=-3 U_{1}-U_{2}+2 U_{3}-U_{5} \\
& U_{1}^{\prime}=U_{3}+2 U_{5} \\
& U_{2}^{\prime}=U_{1}-3 U_{2} \\
& U_{3}^{\prime}=3 U_{1}+U_{2}+4 U_{3}-2 U_{5}
\end{aligned}
$$

The constraints on the singlet vevs obtained by solving Eqs. (6.4b, $)$ are as follows

$$
\begin{aligned}
& x_{45}=\epsilon / 15-\frac{1}{2} V_{3}^{2}, \\
& x_{+}-x_{-}=\epsilon / 15+\frac{1}{2} V_{3}^{2}, \\
& x_{31}-x_{23}=\epsilon / 5, \\
& \sum_{i=3,4}\left(x_{i}\right)+2\left(x_{23}+x_{+}-x_{12}\right)=\bar{V}_{5}^{2}-V_{2}^{2},
\end{aligned}
$$

16 To recover the proper mass units we recall that we have set $\kappa=\sqrt{8 \pi} / M_{P l}=1$ in Eq. (6.4b). 
where $x_{p} \equiv\left|\left\langle\phi_{p}\right\rangle\right|^{2}-\left|\left\langle\bar{\phi}_{p}\right\rangle\right|^{2}$, and $V_{i} \equiv\left\langle\nu_{i}^{c}\right\rangle, \bar{V}_{i} \equiv\left\langle\bar{\nu}_{i}^{c}\right\rangle$. Similarly, $D$-flatness of the anomaly-free $U_{4}(1)$ and $U(1)_{\widetilde{Y}}$ requires

$$
\begin{aligned}
\left|V_{0}\right|^{2} & =\left|V_{1}\right|^{2}+2\left(\left|\eta_{1}\right|^{2}-\left|\bar{\eta}_{1}\right|^{2}+\left|\eta_{2}\right|^{2}-\left|\bar{\eta}_{2}\right|^{2}\right), \\
V^{2} & =\sum_{i=0}^{4}\left|V_{i}\right|^{2}=\left|\bar{V}_{4}\right|^{2}+\left|\bar{V}_{5}\right|^{2}=\bar{V}^{2} .
\end{aligned}
$$

The F-flatness constraints (Eqs. (6.4a)) can also be worked out for the cubic superpotential and the resulting 24 equations (one for each singlet field) can be summarized as follows

$$
\begin{aligned}
& \Phi_{12} \Phi_{31}=\Phi_{12} \Phi_{23}=\phi_{45} \Phi_{3}=0 \quad \text { and h.c., } \\
& \Phi_{23} \Phi_{31}+\phi^{+} \phi^{-}+\phi_{i} \phi_{i}=0 \quad \text { and h.c., } \\
& \phi_{45} \bar{\phi}_{45}+\phi_{i} \bar{\phi}_{i}+\phi^{+} \bar{\phi}^{+}+\phi^{-} \bar{\phi}^{-}=0, \\
& \phi_{3} \bar{\phi}_{4}+\bar{\phi}_{3} \phi_{4}=0, \\
& \phi^{+} \Phi_{3}+\bar{\phi}^{-} \bar{\Phi}_{12}=\phi^{-} \Phi_{3}+\bar{\phi}^{+} \bar{\Phi}_{12}=0 \quad \text { and h.c., } \\
& \frac{1}{2} V_{4} \bar{V}_{4}+\eta_{1} \bar{\eta}_{2}+\bar{\eta}_{1} \bar{\eta}_{2}=0, \\
& \frac{1}{\sqrt{2}} V_{4} \bar{V}_{5}+\bar{\phi}_{3} \Phi_{3}+\bar{\phi}_{4} \Phi_{5}+2 \phi_{3} \Phi_{12}=\phi_{3} \Phi_{3}+\phi_{4} \Phi_{5}+2 \bar{\phi}_{3} \bar{\Phi}_{12}=0, \\
& \bar{\phi}_{4} \Phi_{3}+\bar{\phi}_{3} \Phi_{5}+2 \bar{\phi}_{4} \bar{\Phi}_{12}=0 \quad \text { and h.c. } \\
& \left\{\eta_{1}, \bar{\eta}_{1}, \eta_{2}, \bar{\eta}_{2}\right\} \Phi_{0}=0 .
\end{aligned}
$$

These equations have five classes of solutions when solved in conjunction with the Dflatness constraints in Eqs. (6.7). There are eight possible solutions to Eqs. (6.9a), i.e., $\Phi_{12}=\bar{\Phi}_{12}=\Phi_{3}=0, \Phi_{12}=\bar{\Phi}_{31}=\bar{\Phi}_{23}=\Phi_{3}=0, \Phi_{12}=\bar{\Phi}_{12}=\phi_{45}=\bar{\phi}_{45}=0, \cdots$. Four of these solutions violate either of the D-flatness conditions in Eqs. (6.7b, $)$. The remaining five solutions can be worked out in detail and are as follows:

1. $\Phi_{12}=\bar{\Phi}_{12}=\Phi_{3}=0$,
a. $\Phi_{5}=0, V_{4}=0,66.7$, 6.9b,c,d,f);
b. $\Phi_{5}=0, V_{4} \neq 0, \bar{V}_{5}=0,(6.7),(6.9 b, c, d, f)$;
c. $\Phi_{5} \neq 0, V_{4}=0, \phi_{3,4}=\bar{\phi}_{3,4}=0$, (6.7), (6.9b,c,f)$)$;
d. $\Phi_{5} \neq 0, V_{4} \neq 0, \phi_{3,4}=\bar{\phi}_{3}=0$, (6.7), (6.9b,c,f,g).

2. $\Phi_{12}=\bar{\Phi}_{31}=\bar{\Phi}_{23}=\Phi_{3}=0, \bar{\phi}^{+}=\bar{\phi}^{-}=0, \bar{\phi}_{3,4}=0, \phi_{45} \bar{\phi}_{45}=0, V_{4}=0, \bar{V}_{5}^{2}>V_{2}^{2}$,

a. $\Phi_{5}=0,(6.7),(6.9 b, f)$;

b. $\Phi_{5} \neq 0, \phi_{3,4}=0$, (6.7), (6.9b,fl). 
3. $\bar{\Phi}_{12}=\Phi_{31}=\Phi_{23}=\Phi_{3}=0, \phi^{+}=\phi^{-}=0, \phi_{3,4}=0, \phi_{45} \bar{\phi}_{45}=0, \bar{V}_{5}^{2}<V_{2}^{2}$,

a. $\Phi_{5}=0,(6.7),(6.9 b, f, g)$;

b. $\Phi_{5} \neq 0, \bar{\phi}_{3}=0,6.7$, 6.9b,f,g).

4. $\Phi_{12}=\bar{\Phi}_{31}=\bar{\Phi}_{23}=0, \phi_{45}=\bar{\phi}_{45}=0, \bar{\phi}^{+}=\bar{\phi}^{-}=0, V_{3}^{2}=2 \epsilon / 15$,

a. $\Phi_{5}=0, \phi_{3,4}=\bar{\phi}_{3,4}=0, \bar{V}_{5}^{2}>V_{2}^{2}, 6.7$, 6.9b,f,g);

b. $\Phi_{5} \neq 0,(6.7),(6.9 b, c, d, f, g, h)$.

5. $\bar{\Phi}_{12}=\Phi_{31}=\Phi_{23}=0, \phi_{45}=\bar{\phi}_{45}=0, \phi^{+}=\phi^{-}=0, V_{3}^{2}=2 \epsilon / 15$,

a. $\Phi_{5}=0, \phi_{3,4}=\bar{\phi}_{4}=0, \bar{V}_{5}^{2}<V_{2}^{2},(6.7),(6.9 b, f, g)$;

b. $\Phi_{5} \neq 0,(\underline{6.7}),(6.9 b, c, d, f, g, h)$.

In cases 2 and 3 , the solution to $\phi_{45} \bar{\phi}_{45}=0$ depends on the sign of $\xi=\epsilon / 15-\frac{1}{2} V_{3}^{2}$. For $\xi>0(<0), \bar{\phi}_{45}=0\left(\phi_{45}=0\right)$, whereas for $\xi=0, \phi_{45}=\bar{\phi}_{45}=0$. Note also that cases 2 and 3 are mutually exclusive. In solving Eqs. (6.9i), we have assumed that $\eta_{1,2}, \bar{\eta}_{1,2}$ have generically nonvanishing vevs and therefore $\Phi_{0}=0$. If $\eta_{1,2}=\bar{\eta}_{1,2}=0$, further simplification of the above solutions is possible.

\subsection{Symmetry breaking and EVA mechanism}

There are five high-energy scales in this problem, which are correlated and in principle self-consistently determined, as follows:

(i) the string unification scale $M_{U} \approx 7.3 \times g \times 10^{17} \mathrm{GeV}$ [49,30,31;

(ii) the scale of $U_{A}$ anomaly cancellation vevs $\langle\phi\rangle \sim \sqrt{\epsilon}=\mathcal{O}\left(10^{17} \mathrm{GeV}\right)$;

(iii) the $S U(5) \times U(1)_{\widetilde{Y}} \rightarrow S U(3)_{C} \times S U(2)_{L} \times U(1)_{Y}$ symmetry breaking scale $M_{X}$, undetermined theoretically but phenomenologically of $\mathcal{O}\left(10^{15-18} \mathrm{GeV}\right)$;

(iv) the scale where the surplus $Q, \bar{Q}$ get heavy $M_{\text {eva }}$ (the EVA scale [50]), to be determined;

(v) the scales of hidden sector condensation $\Lambda_{4}$ and $\Lambda_{10}$, to be determined from the hidden sector matter spectrum.

The gauge symmetry breaking occurs as follows

$$
\begin{aligned}
S U(5) \times U(1)_{\widetilde{Y}} \times U_{4}(1) & \supset S U(3)_{C} \times S U(2)_{L} \times U(1)_{Y^{\prime}} \times U(1)_{\widetilde{Y}} \times U_{4}(1) \\
& \rightarrow S U(3)_{C} \times S U(2)_{L} \times U(1)_{Y},
\end{aligned}
$$

where we have assumed that $U_{4}(1)$ remains unbroken, i.e., $\eta_{1,2}=\bar{\eta}_{1,2}=0$. This appears to be a reasonable choice since these fields do not participate in the anomalous 
$U_{A}(1)$ cancellation. Also, if $\eta_{1,2}, \bar{\eta}_{1,2} \neq 0$, the subsequent discussion will remain qualitatively unaffected. We will generally assume that $V_{0}, V_{1}, \bar{V}_{5} \neq 0, V_{4}=0$, and optionally $V_{2,3}, \bar{V}_{4} \neq 0$. Given the charges of $\nu_{0,1}^{c}, \bar{\nu}_{5}^{c}$ under the relevant $U(1)$ symmetries, it is clear that only $Y=\frac{1}{5} Y^{\prime}-\frac{2}{5} \widetilde{Y}$ remains unbroken below the scale $M_{X}$. In fact, there are two flat directions of the scalar potential: $\varphi_{1}=\frac{1}{2}\left(\nu_{0}^{c}+\nu_{1}^{c}\right)+\frac{1}{\sqrt{2}} \bar{\nu}_{5}^{c}$ and $\varphi_{2}=\frac{1}{\sqrt{2}}\left(\nu_{0}^{c}+\nu_{1}^{c}\right)$. The imaginary parts of the scalar components of these fields are eaten by the broken $U(1)$ gauge bosons and the remaining supermultiplet components become heavy higgs/higgsino states. There is another linearly independent field which remains light, the so-called flaton/flatino supermultiplet [51]. The $d_{0,1}^{c}, \bar{d}_{5}^{c}$ components of $F_{0,1}, \bar{F}_{5}$ appear in the higgs triplet mass matrix (see Subsec. 6.4). We are then left with the $Q_{0,1}, \bar{Q}_{4,5}$ components. The scalars get either eaten by the $X, Y$ gauge bosons or become heavy higgs bosons, whereas the fermions interact with the $\tilde{X}, \tilde{Y}$ gauginos through the following mass matrix

$$
\begin{array}{r}
\mathcal{M}_{1 / 2}= \\
\bar{Q}_{0} \\
Q_{1} \\
\widetilde{X}
\end{array}\left(\begin{array}{ccc}
w_{0}^{(4)} & \bar{Q}_{5} & \tilde{Y} \\
w_{1}^{(5)} & w_{5} V_{0} \\
g_{5} \bar{V}_{4} & g_{5} \bar{V}_{5} & g_{5} V_{1}
\end{array}\right),
$$

where $w_{0,1}^{(4,5)}$ come from higher-order superpotential couplings, and effect the EVA mechanism. We expect all these fields to become heavy, although at different mass scales (see Sec. 7).

\subsection{The Higgs mass matrices}

The Higgs doublet masses originate from the $h_{i} \bar{h}_{j} \rightarrow H_{i} \bar{H}_{j}$ and $F_{i} \bar{f}_{j} \bar{h}_{k} \rightarrow V_{i} L_{j} \bar{H}_{k}$ couplings and to quartic order are

$$
\begin{aligned}
& \begin{array}{llll}
\bar{H}_{1} & \bar{H}_{2} & \bar{H}_{3} & \bar{H}_{45}
\end{array}
\end{aligned}
$$

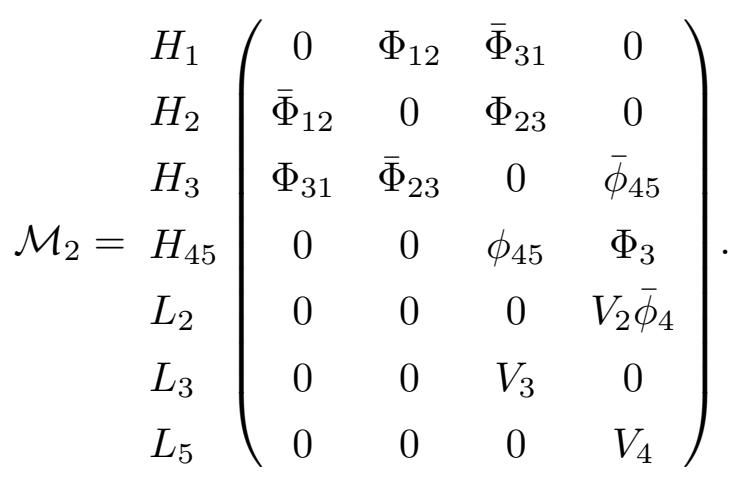


The Higgs triplet mass matrix which effects the doublet/triplet splitting receives contributions from: $h_{i} \bar{h}_{j} \rightarrow D_{i} \bar{D}_{j}, F_{i} F_{j} h_{k} \rightarrow V_{i} D_{k} d_{j}^{c}, \bar{F}_{i} \bar{F}_{j} \bar{h}_{k} \rightarrow \bar{V}_{i} \bar{d}_{j}^{c} \bar{D}_{k}$, and $F_{i} \bar{F}_{j} \rightarrow \bar{d}_{j}^{c} d_{i}^{c}$. The resulting matrix is

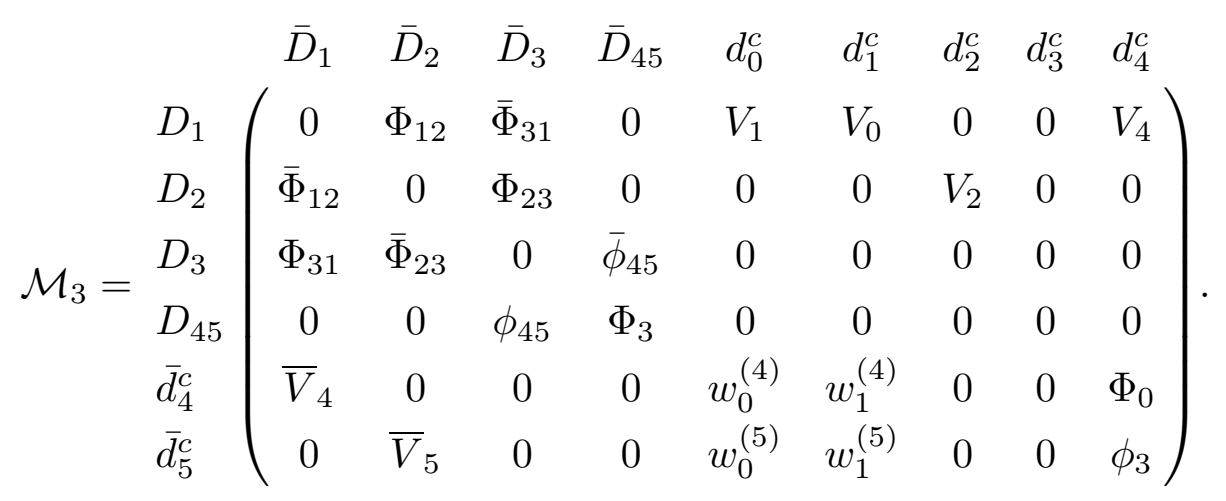

\subsection{A possible numerical scenario}

Until the full set of quintic (and possibly higher-order) superpotential couplings is calculated, one cannot work out some features of the model such as the precise eigenvalues of the doublet and triplet mass matrices, or the diagrams contributing to proton decay, or the details of gauge coupling unification in this model. Nevertheless, we can postulate a possible scenario (to be confirmed by further calculations) given the limited amount of information available at this time.

There are three basic phenomenological considerations which help decide on the vevs left undetermined by the flatness conditions: (i) dimension-five operators in proton decay, (ii) the fermion Yukawa couplings and the higgs doublet mass matrix, and (iii) the higgs triplet mass matrix. Cubic couplings of the form $F_{a} F_{b} h \supset Q_{a} Q_{b} D, F_{c} \bar{f} \bar{h} \supset Q_{c} L \bar{D}$, $h \bar{h} \phi \supset D \bar{D} \phi$ would be disastrous for proton decay [26]. We have (see Eq. (6.3a)): $F_{0} F_{1} h_{1}, F_{2} F_{2} h_{2}, F_{4} F_{4} h_{1} ; F_{4} \bar{f}_{5} \bar{h}_{45}, F_{3} \bar{f}_{3} \bar{h}_{3} ; h_{1} \bar{h}_{3} \bar{\Phi}_{31}, h_{2} \bar{h}_{3} \Phi_{23}$. With our assumptions in Sec. $6.3, Q_{0,1}$ do not contain quark fields, whereas $Q_{2,3,4}$ do. It is then important to have $\Phi_{23}=0$, and perhaps also $\bar{\Phi}_{31}=0$, assuming $Q_{2}\left(Q_{4}\right)$ contains second (third) generation quark fields. It turns out that these two constraints are either automatic or can be imposed (and still maintain flatness) in the five $F$-flatness cases in Sec. 6.2. In case 1 both $H_{1}$ and $H_{2}$ become pure massless states (see Eq. (6.12)), whereas in cases 2 and 4 (3 and 5) $H_{1}$ $\left(H_{2}\right)$ is a pure massless state. Based on the sensible assumption that (so far neglected) higher-order contributions to $\mathcal{M}_{2}$ will likely make massive (light) mixed states rather than (light) pure states (since the former have more possible couplings), we conclude that cases 2 and 4 are the most promising ones, since the others would give too many cubic fermion Yukawa couplings. 
Let us analyze the two preferred cases in turn:

(a) Case 2. We need to impose $\Phi_{23}=0$, while we get $\bar{\Phi}_{31}=0$ automatically. To avoid $\bar{H}_{45}$ becoming heavy, we need the $\bar{\phi}_{45}=0$ solution to $\phi_{45} \bar{\phi}_{45}=0$ (which assumes that $\xi=\epsilon / 15-\frac{1}{2} V_{3}^{2}>0$, and is then consistent with the choice $\left.V_{3}=0\right)$ and $V_{2}=0$ (also consistent with $\bar{V}_{5}^{2}>V_{2}^{2}$ ). We then get $H_{1}, H_{23 \ell} \propto-\left\langle\Phi_{31}\right\rangle H_{2}+\left\langle\bar{\Phi}_{12}\right\rangle H_{3}$, $L_{2,3,5} ; \bar{H}_{2}, \bar{H}_{45}$ light and $H_{23 h} \propto\left\langle\bar{\Phi}_{12}\right\rangle H_{2}+\left\langle\Phi_{31}\right\rangle H_{3}, H_{45} ; \bar{H}_{1}, \bar{H}_{3}$ heavy. We expect higher-order contributions to $\mathcal{M}_{2}$ to give an intermediate scale mass to one pair of the remaining light doublets. The cubic fermion Yukawa couplings which remain are then $F_{4} \bar{f}_{5} \bar{h}_{45} \supset Q_{4} u_{4}^{c} \bar{H}_{45}$ and $F_{4} F_{4} h_{1} \supset Q_{4} d_{4}^{c} H_{1}$, leading to the identification $\lambda_{t}=\lambda_{b}=g \sqrt{2}$. At the quartic level we have (see Eq. (6.3b)) $c F_{2} \bar{f}_{2} \bar{h}_{45} \bar{\phi}_{4}$ which will give a vanishing coupling since $\bar{\phi}_{4}=0$ in this case. Preliminary probing into the quintic couplings reveals a structure of the form

$$
\begin{aligned}
& F_{2} F_{2} h_{1}\left\{\bar{\phi}^{+} \bar{\phi}^{-}, \bar{\phi}_{3} \bar{\phi}_{3}, \bar{\phi}_{4} \bar{\phi}_{4}\right\}, \\
& \bar{f}_{2} l_{2}^{c} h_{1}\left\{\bar{\phi}^{+} \bar{\phi}^{-}, \bar{\phi}_{3} \bar{\phi}_{3}, \bar{\phi}_{4} \bar{\phi}_{4}\right\}, \\
& \bar{f}_{5} l_{5}^{c} h_{1}\left\{\bar{\phi}_{3} \bar{\phi}_{3}, \bar{\phi}_{4} \bar{\phi}_{4}\right\} .
\end{aligned}
$$

All these couplings will also vanish since $\bar{\phi}^{+}=\bar{\phi}^{-}=\bar{\phi}_{3,4}=0$ in this case. This scenario appears disfavored.

(b) Case 4. Here we also need to impose $\Phi_{23}=0$, whereas $\bar{\Phi}_{31}=0$ is automatic. To obtain a light $\bar{H}_{45}$ we need to set $\Phi_{3}=V_{2}=V_{4}=0$. However, $V_{3}^{2}=2 \epsilon / 15>0$ in this case and the higgs doublet mass matrix has a novel structure. We get $H_{1}, H_{45}, H_{23 \ell}$, $L_{2,5} ; \bar{H}_{2}, \bar{H}_{45}$ light, and $H_{23 h}, L_{3} ; \bar{H}_{1}, \bar{H}_{3}$ heavy. Note that there will be a mixing between "higgs" and "lepton" doublets in this case, since we expect one pair of the remaining light doublets to become heavy when higher-order contributions to $\mathcal{M}_{2}$ are included. The advantage of this case over Case 2 above is that the vevs contributing to the higher-order couplings are generally non-zero. We obtain: $\lambda_{t}=\lambda_{b}=g \sqrt{2}$, $\lambda_{c}=c\left\langle\bar{\phi}_{4}\right\rangle / M$, with $c=\mathcal{O}(1)$ and $\langle\phi\rangle / M \lesssim 1 / 10$ [24]. Of the three quintic couplings in Eq. (6.14), the first of these could give the strange-quark Yukawa coupling, the second the tau Yukawa, and the third one the $\mu$ Yukawa. For this assignment to be realistic we would need the second coupling $\left(\lambda_{\tau}\right)$ to be sizeable despite its potential suppression by $\langle\phi\rangle / M$, whereas the first coupling should remain small (and so should the third one). These details may be possible to arrange since there are three sources of uncertainty at this point: the actual coefficient of the terms, the size of the singlet 
vevs involved, and the possibility of mixing between $Q_{2}\left(d_{2}^{c}\right)$ and some of the other $Q$ 's ( $d^{c}$ s) in the symmetry breaking process (higgs triplet mass matrix).

A study of the triplet mass matrix $\mathcal{M}_{3}$ is not very illuminating at this time, although it is clear that we must demand $V_{4}=\Phi_{0}=\phi_{3}=0$ to obtain a light $d_{4}^{c}$. It is also clear that both $d_{0,1}^{c}$ become heavy. In case $4, \bar{D}_{1,2} ; D_{1}, \bar{d}_{5}^{c}$, and a linear combination of $D_{2}, D_{3}, \bar{d}_{4}^{c}$ are also heavy. Higher-order contributions to $\mathcal{M}_{3}$ are needed to continue this analysis further.

\section{Gauge coupling unification}

Let us now address the question which motivated our extensive search for a $5 / 2$ model. If one starts from the low-energy values of the gauge couplings $\left(\sin ^{2} \theta_{w}=0.2331 \pm 0.0013\right.$ [52], $\alpha_{3}=0.113 \pm 0.004$ [53], and $\alpha_{e}^{-1}=127.9 \pm 0.2$ ) and expects to get gauge coupling unification at a scale $M_{U}$, then various intermediate-scale particles have to contribute in suitable ways to the running of the gauge couplings. The $S U(5) \times U(1)_{\widetilde{Y}}$ breaking scale $M_{X}$ is an unknown in the problem, but not the only one since (as discussed in Sec. 6.3) there are other scales inter-related to it. As an example we could imagine that $M_{X}$ is close to $M_{U}$, in which case the expressions of Refs. [32, 37] apply (for $M_{X}<M_{U}$ see Ref. [36]), as follows

$$
\begin{aligned}
L_{Q} & =(23.31-25.86)+\frac{1}{2} L_{U^{c}}+\frac{1}{2} L_{E^{c}}+2 L_{\widetilde{F}}, \\
L_{D^{c}} & =(58.29-67.90)+L_{L}+\frac{1}{2} L_{E^{c}}+2 L_{\widetilde{F}}-\frac{1}{2} L_{U^{c}}
\end{aligned}
$$

where $L_{R}=\sum_{i} \ln \left(M_{U} / m_{R_{i}}\right)$ and the sum runs over all the supermultiplets in representation $R_{i}$. The constant ranges allow for values of the low-energy parameters inside the 1- $\sigma$ error ellipsoid [37]. In the present model there are no $U^{c}$ or $E^{c}$ representations besides the standard ones, thus $L_{U^{c}}=L_{E^{c}}=0$ in (7.1). The important point encoded in these expressions is the fact that $L_{Q}>0$ and therefore intermediate-scale extra vector-like $Q$ representations are needed. These are not present in 4/1 models, and one pair is present in $5 / 2$ models. Note also that $L_{D^{c}}>0\left(\right.$ since $\left.L_{U^{c}}=0\right)$ and we must also have extra vector-like $D^{c}$ representations at intermediate mass scales, but this is always the case in flipped $S U(5)$ models.

From Table IV and the vev choices made in Sec. 6, the contribution to the running of the gauge couplings can be split up into five groups:

1. $F_{2,3,4}, \bar{f}_{2,3,5}, l_{2,3,5}^{c}$ contribute the usual three generations of quarks and leptons. 
2. $H_{1,2,3,45}, \bar{H}_{1,2,3,45}$ contribute to $L_{L}=\sum_{i} \ln \left(M_{U} / m_{i}^{(2)}\right)^{2}$, where $m_{i}^{(2)}$ are the eigenvalues of $\mathcal{M}_{2} \mathcal{M}_{2}^{T}$. (Note that $L_{L}$ includes the contribution from the expected two light higgs doublets.)

3. $D_{1,2,3,45}, \bar{d}_{4,5}^{c} ; \bar{D}_{1,2,3,45}, d_{0,1}^{c}$ contribute to $L_{D^{c}}=\sum_{i} \ln \left(M_{U} / m_{i}^{(3)}\right)^{2}$, where $m_{i}^{(3)}$ are the eigenvalues of $\mathcal{M}_{3} \mathcal{M}_{3}^{T}$ with the last three columns removed from $\mathcal{M}_{3}$.

4. $Q_{0}, Q_{1} \rightarrow Q_{H}=\left(Q_{0}+Q_{1}\right) / \sqrt{2}, Q_{\text {eva }}=\left(Q_{0}-Q_{1}\right) / \sqrt{2}$ and $\bar{Q}_{4}, \bar{Q}_{5} \rightarrow \bar{Q}_{H}=\left(\bar{V}_{4} \bar{Q}_{4}+\right.$ $\left.\bar{V}_{5} \bar{Q}_{5}\right) / \bar{V}, \bar{Q}_{\text {eva }}=\left(\bar{V}_{5} \bar{Q}_{4}-\bar{V}_{4} \bar{Q}_{5}\right) / \bar{V}$, where $Q_{H}, \bar{Q}_{H}$ become heavy higgs/higgsino states in the $S U(5) \times U(1)_{\widetilde{Y}}$ symmetry breaking process (see Eq. (6.11) with $w \ll$ $V, \bar{V}$ ) and $Q_{e v a}, \bar{Q}_{e v a}$ get masses $M_{e v a}=\mathcal{O}(w)$. (Note that in Eq. (6.11) we need $w_{0}^{(4)} \neq w_{1}^{(4)}$ or $w_{0}^{(5)} \neq w_{1}^{(5)}$ to avoid a massless $Q_{e v a}, \bar{Q}_{\text {eva }}$ pair.) The latter contribute to $L_{Q}=2 \ln \left(M_{U} / M_{\text {eva }}\right)$.

5. $\widetilde{F}_{i}, \widetilde{\bar{F}}_{j}$ which have $\pm \frac{1}{2}$ electric charges contribute to $L_{\widetilde{F}}=\sum_{i} \ln \left(M_{U} / m_{i}^{( \pm 1 / 2)}\right)^{2}$ where $m_{i}^{( \pm 1 / 2)}$ are the eigenvalues of the mass matrix $\mathcal{M}_{\widetilde{F}} \mathcal{M}_{\widetilde{F}}^{T}$ with $\widetilde{F}_{i} \mathcal{M}_{\widetilde{F}} \widetilde{\bar{F}}_{j}$.

From Eq. (7.10) it is clear that $L_{Q} \geq(23.31-25.86)$ since $L_{\widetilde{F}} \geq 0$ (recall that $L_{U^{c}}=$ $\left.L_{E^{c}}=0\right)$. From the expression for $L_{Q}$ just derived, it follows that $M_{\text {eva }} \lesssim 10^{12} \mathrm{GeV}$. This upper bound is reduced to $\approx 10^{10,8,6} \mathrm{GeV}$ for $M_{X}=10^{17,16,15} \mathrm{GeV}$ [36]. This is the only direct test of gauge coupling unification in this model which we can perform at this time since it only involves the determination of the scale $M_{\text {eva }}$. We have explored the quintic terms in the superpotential and found terms of the form $F_{0,1} \bar{F}_{4,5}\langle\phi\rangle\langle D D, \widetilde{F} \widetilde{\bar{F}}\rangle / M^{2}$ which would give $w \sim M_{\text {eva }} \sim \mathcal{O}\left(10^{5} \mathrm{GeV}\right)$ if the $S U(4)_{h}$ condensation scale is $\mathcal{O}\left(10^{12} \mathrm{GeV}\right)$ and $\langle\phi\rangle \sim 10^{17} \mathrm{GeV}$. These are plausible numbers which do not violate the upper bound on $M_{\text {eva }}$ even for $M_{X}=10^{15} \mathrm{GeV}$, and still leave room for a non-vanishing $L_{\widetilde{F}}$.

\section{Conclusions}

Gauge coupling unification is one of the few universal predictions of string models (together with the presence of gravity and gauge interactions). An even more pervasive fact is that the string unification scale can be precisely calculated in any given string model. The robustness of this prediction is in sharp constrast with the basically unlimited number of possible models with or without supersymmetry, and with all possible gauge groups and matter representations. (The space of models possesses certain internal structure which for example correlates gauge groups and matter representations.) Nevertheless, string unification in any given model can actually be tested. Indeed, any such model gives in principle definite predictions for some of the best measured parameters at the electroweak 
scale, namely $\sin ^{2} \theta_{w}$ and $\alpha_{3}$. From this point of view, traditional unified models are also testable this way [33], although there the unification scale has to be chosen to fit the experimental data.

The motivation for this paper was to search for a flipped $S U(5)$ model which could possibly accommodate the measured values of the low-energy gauge couplings or equivalently which could unify at the string scale. There is a necessary condition for such a model (in level-one Kac-Moody constructions and with small string threshold effects), that it possesses extra $Q, \bar{Q}$ representations, i.e., a 5/2 model. To pursue this objective within the free fermionic formulation, we have developed a sophisticated and comprehensive computer program which can sweep systematically over large numbers of models and determine their gauge group, massless spectrum, and cubic superpotential, and decide to keep only "interesting" models. Our search space, even though vast, is by no means exhaustive and much of it still remains uncharted. Nevertheless, the structure of what we did explore has proved to be very simple. Of the few supersymmetric $5 / 2$ models which we found, only one of them allows for both top- and bottom-quark Yukawa couplings. Further probe into the superpotential and the $D$ - and $F$-flatness constraints indicates that the model is phenomenologically sound. However, much work remains to be done to bring this model up to the level of development of its predecessor (the revamped model). An encouraging result

is that the masses of the extra $Q, \bar{Q}$ states are likely to fall within the bounds imposed by string unification.

Contrary to popular belief, string models can be tested. Moreover, if models which satisfy all known phenomenological constraints can ever be found, then they will become candidates for the theory of everything until they are defeated by future tests or until string theory hands down its final verdict, whichever comes first.

\section{Acknowledgments}

We would like to thank I. Antoniadis and J. Ellis for useful discussions at the earlier stages of this work. This work has been supported in part by DOE grant DE-FG05-91ER-40633. The work of K.Y has been supported in part by the Texas National Laboratory Research Commission under Grant No. RCFY9155, and in part by the U.S. Department of Energy under Grant No. DE-FG05-84ER40141. 


\section{References}

[1] See e.g., String theory in four dimensions, ed. by M. Dine, (North-Holland, Amsterdam, 1988); Superstring construction, ed. by A.N. Schellekens (North-Holland, Amsterdam, 1989).

[2] I. Antoniadis, C. Bachas, and C. Kounnas, Nucl. Phys. B 289 (1987) 87.

[3] I. Antoniadis and C. Bachas, Nucl. Phys. B 298 (1988) 586.

[4] H. Kawai, D.C. Lewellen, and S.-H. Tye, Phys. Rev. Lett. 57 (1986) 1832; Phys. Rev. D 34 (1986) 3794; Nucl. Phys. B 288 (1987) 1.

[5] H. Kawai, D.C. Lewellen, J.A. Schwarz, and S.-H.H. Tye, Nucl. Phys. B 299 (1988) 431.

[6] R. Bluhm, L. Dolan, and P. Goddard, Nucl. Phys. B 309 (1988) 330.

[7] H. Dreiner, J.L. Lopez, D.V. Nanopoulos, and D. Reiss, Nucl. Phys. B 320 (1989) 401.

[8] S. Kalara, J. Lopez, and D.V. Nanopoulos, Phys. Lett. B 245 (1990) 421, Nucl. Phys. В 353 (1991) 650.

[9] For a review see e.g., P. Goddard and D. Olive, Int. J. Mod. Phys. A 1 (1986) 303.

[10] J. Ellis, J. Lopez, and D.V. Nanopoulos, Phys. Lett. B 245 (1990) 375.

[11] A. Font, L. Ibáñez, and F. Quevedo, Nucl. Phys. B 345 (1990) 389.

[12] D. Lewellen, Nucl. Phys. B 337 (1990) 61; J. A. Schwarz, Phys. Rev. D 42 (1990) 1777.

[13] S. Barr, Phys. Lett. B 112 (1982) 219, Phys. Rev. D 40 (1989) 2457; J. Derendinger, J. Kim, and D.V. Nanopoulos, Phys. Lett. B 139 (1984) 170.

[14] I. Antoniadis, J. Ellis, J. Hagelin, and D.V. Nanopoulos, Phys. Lett. B 194 (1987) 231.

[15] For a recent review see e.g., J.L. Lopez and D.V. Nanopoulos, Texas A \& M University preprint CTP-TAMU-76/91, to appear in Proceedings of the 15th Johns Hopkins Workshop on Current Problems in Particle Theory, August 1991.

[16] I. Antoniadis, G. Leontaris, and J. Rizos, Phys. Lett. B 245 (1990) 161.

[17] B. Greene, K.H. Kirklin, P.J. Miron, and G.G. Ross, Phys. Lett. B 180 (1986) 69; Nucl. Phys. B 278 (1986) 667; Nucl. Phys. B 292 (1987) 606; S. Kalara and R.N. Mohapatra, Phys. Rev. D 36 (1987) 3474; J. Ellis, K. Enqvist, D.V. Nanopoulos, and K. Olive, Nucl. Phys. B 297 (1988) 103; R. Arnowitt and P. Nath, Phys. Rev. D 39 (1989) 2006.

[18] L. Ibáñez, H. Nilles, and F. Quevedo, Phys. Lett. B 187 (1987) 25; L. Ibáñez, J. Mas, H. Nilles, and F. Quevedo, Nucl. Phys. B 301 (1988) 157; A. Font, L. Ibáñez, F. Quevedo, and A. Sierra, Nucl. Phys. B 331 (1990) 421. 
[19] A. Faraggi, D.V. Nanopoulos, and K. Yuan, Nucl. Phys. B 335 (1990) 347; A. Faraggi, Texas A \& M University preprint CTP-TAMU-49/91; K. Yuan, PhD Thesis Texas A\&M University (1991).

[20] I. Antoniadis, J. Ellis, J. Hagelin, and D.V. Nanopoulos, Phys. Lett. B 231 (1989) 65.

[21] I. Antoniadis, J. Ellis, J. Hagelin, and D.V. Nanopoulos, Phys. Lett. B 205 (1988) 459.

[22] I. Antoniadis, J. Ellis, J. Hagelin, and D.V. Nanopoulos, Phys. Lett. B 208 (1988) 209.

[23] T.T. Burwick, R.K. Kaiser, and H.F. Muller, Nucl. Phys. B 362 (1991) 232; D. Bailin, E.K. Katechou, and A. Love, Int. J. Mod. Phys. A 7 (1992) 153.

[24] J. L. Lopez and D.V. Nanopoulos, Phys. Lett. B 251 (1990) 73 and Phys. Lett. B 268 (1991) 359.

[25] J. Ellis, J. Lopez, and D.V. Nanopoulos, Phys. Lett. B 247 (1990) 257.

[26] J. Ellis, J.L. Lopez, and D.V. Nanopoulos, Phys. Lett. B 252 (1990) 53; G. Leontaris and K. Tamvakis, Phys. Lett. B 260 (1991) 333.

[27] J. Rizos and K. Tamvakis, Phys. Lett. B 251 (1990) 369; I. Antoniadis, J. Rizos, and K. Tamvakis, Ecole Polytechnique preprint CPTH-A092.1191 (1991).

[28] J. L. Lopez and D.V. Nanopoulos, Phys. Lett. B 256 (1991) 150; S. Kalara, J.L. Lopez, and D.V. Nanopoulos, Phys. Lett. B 275 (1992) 304 and Texas A \& M University preprint CTP-TAMU-94/91.

[29] G. Leontaris, Phys. Lett. B 207 (1988) 447; G. Leontaris and D.V. Nanopoulos, Phys. Lett. B 212 (1988) 327; G. Leontaris and K. Tamvakis, Phys. Lett. B 224 (1989) 319; S. Abel, Phys. Lett. B 234 (1990) 113; I. Antoniadis, J. Rizos, and K. Tamvakis, Ecole Polytechnique preprint CPTH-A140.0192 (1992).

[30] I. Antoniadis, J. Ellis, R. Lacaze, and D.V. Nanopoulos, Phys. Lett. B 268 (1991) 188.

[31] S. Kalara, J.L. Lopez, and D.V. Nanopoulos, Phys. Lett. B 269 (1991) 84.

[32] I. Antoniadis, J. Ellis, S. Kelley, and D.V. Nanopoulos, Phys. Lett. B 272 (1991) 31.

[33] J. Ellis, S. Kelley and D. V. Nanopoulos, Phys. Lett. B 249 (1990) 441.

[34] L. Ibáñez, D. Lüst, and G. Ross, Phys. Lett. B 272 (1991) 251; L. Ibáñez and D. Lüst, CERN preprint CERN-TH.6380 (1992).

[35] D. Bailin and A. Love, Sussex preprint SUSX-TH-91/16.

[36] D. Bailin and A. Love, Sussex preprint SUSX-TH-91/17.

[37] S. Kelley, J.L. Lopez, and D.V. Nanopoulos, Texas A \& M University preprint CTPTAMU-84/91 (to appear in Phys. Lett. B).

[38] N. Seiberg and E. Witten, Nucl. Phys. B 276 (1986) 272; L. Alvarez-Gaumé, G. Moore and C. Vafa, Comm. Math. Phys. 106 (1986) 1.

[39] I. Antoniadis, C. Bachas, C. Kounnas, and P. Windey, Phys. Lett. B 171 (1986) 51.

[40] F. Gliozzi, J. Scherk and D. Olive, Nucl. Phys. B 122 (1977) 253. 
[41] D. Senechal, Phys. Rev. D 39 (1989) 3717.

[42] L. Dixon, V. Kaplunovsky, and C. Vafa, Nucl. Phys. B 294 (1987) 43; T. Banks, L. Dixon, D. Friedan, and E. Martinec, Nucl. Phys. B 299 (1988) 364.

[43] See e.g., R. Gilmore, Lie Groups, Lie Algebras, and Some of Their Applications (John Wiley \& Sons, New York, 1974).

[44] See e.g., J. Humphreys, Introduction to Lie Algebras and Representation Theory (Springer Verlag, New York, 1980).

[45] R. Slansky, Phys. Rep. 79 (1981) 1.

[46] J. Banks and H. Georgi, Phys. Rev. D 14 (1976) 1159.

[47] A. Faraggi and D.V. Nanopoulos, Texas A \& M University preprint CTP-TAMU-78/90 (unpublished).

[48] J.L. Lopez and D.V. Nanopoulos, Nucl. Phys. B 338 (1990) 73.

[49] V. Kaplunovsky, Nucl. Phys. B 307 (1988) 145; J. Derendinger, S. Ferrara, C. Kounnas, and F. Zwirner, CERN preprint CERN-TH.6004/91 (revised version).

[50] S. Kelley, J.L. Lopez, and D.V. Nanopoulos, Phys. Lett. B 261 (1991) 424.

[51] J. Ellis, J. Hagelin, S. Kelley, D.V. Nanopoulos, and K. Olive, Phys. Lett. B 209 (1988) 283.

[52] J. Ellis, S. Kelley, and D.V. Nanopoulos, CERN preprint CERN-TH.6140/91.

[53] J. Ellis, D.V. Nanopoulos, and D. Ross, Phys. Lett. B 267 (1991) 132. 
Table I: The rank and determinant 44] of the Cartan matrices $C$ of all the Lie algebras. As usual, $A_{l}=S U(l+1), B_{l}=S O(2 l+1), C_{l}=S p(2 l), D_{l}=S O(2 l)$.

\begin{tabular}{|c|c|c|}
\hline Algebra & $\operatorname{det} C$ & rank \\
\hline$A_{l}$ & $l+1$ & $l$ \\
$B_{l}$ & 2 & $l$ \\
$C_{l}$ & 2 & $l$ \\
$D_{l}$ & 4 & $l$ \\
$E_{6}$ & 3 & 6 \\
$E_{7}$ & 2 & 7 \\
$E_{8}$ & 1 & 8 \\
$F_{4}$ & 1 & 4 \\
$G_{2}$ & 1 & 2 \\
\hline
\end{tabular}

Table II: The set of spin-structure basis vectors which are common in the construction of all our models. The first entry corresponds to the complexified $\psi^{\mu}$ and the next 18 to the six left-moving triplets $\left(\chi^{\ell}, y^{\ell}, \omega^{\ell}\right)$. The first 12 right-moving entries (to the right of the colon) correspond to the real fermions $\bar{y}^{\ell}, \bar{\omega}^{\ell}$, and the last 16 correspond to complex fermions. A 1 (0) stands for periodic (antiperiodic) boundary conditions. We also use the symbols $1_{8}=11111111$ and $0_{8}=00000000$.

$$
\begin{aligned}
& \mathbf{1}=\left(\begin{array}{l}
1111111111111111111 \text { : } 111111111111111111111_{8}
\end{array}\right) \\
& S=\left(\begin{array}{l}
1100100100100100100: 0000000000000000000000
\end{array}\right)
\end{aligned}
$$

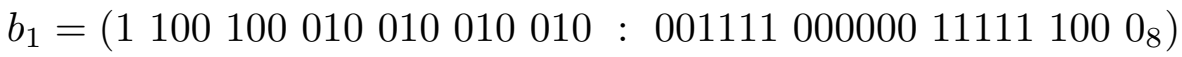

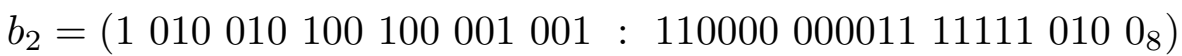

$$
\begin{aligned}
& b_{3}=\left(\begin{array}{ll}
1001001001001100100: 0000001111001111100100 & 0
\end{array}\right) \\
& b_{4}=\left(\begin{array}{l}
1100100010001001010 \quad: 0010010001101111110008
\end{array}\right)
\end{aligned}
$$

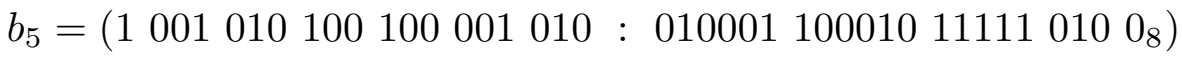


Table III: Classification of $\alpha$-vectors according to the number and type of residual $U_{a, b, c}$ gauge symmetries which they break or do not break. $U_{a}: \bar{w}^{2} \bar{w}^{3}, U_{b}: \bar{y}^{1} \bar{w}^{6}$, and $U_{c}: \bar{y}^{4} \bar{y}^{5}$.

\begin{tabular}{|c|c|c|c|c|}
\hline Class & rank & $U_{a}$ & $U_{b}$ & $U_{c}$ \\
\hline 1 & 19 & $\sqrt{ }$ & $\sqrt{ }$ & $\sqrt{ }$ \\
$2 \mathrm{a}$ & 18 & $\sqrt{ }$ & $\sqrt{ }$ & $\times$ \\
$2 \mathrm{~b}$ & 18 & $\sqrt{ }$ & $\times$ & $\sqrt{ }$ \\
$2 \mathrm{c}$ & 18 & $\times$ & $\sqrt{ }$ & $\sqrt{ }$ \\
$3 \mathrm{a}$ & 17 & $\sqrt{ }$ & $\times$ & $\times$ \\
$3 \mathrm{~b}$ & 17 & $\times$ & $\sqrt{ }$ & $\times$ \\
$3 \mathrm{c}$ & 17 & $\times$ & $\times$ & $\sqrt{ }$ \\
4 & 16 & $\times$ & $\times$ & $\times$ \\
\hline
\end{tabular}


Table IV: The massless spectrum of the selected 5/2 model with gauge group $S U(5) \times$ $U(1)_{\widetilde{Y}} \times S O(10)_{h} \times S U(4)_{h} \times U(1)^{5}$ and $2 / 3 / 2$ Yukawa set. The transformation properties of the observable sector fields under $S U(5) \times U(1)_{\widetilde{Y}}$ are as follows: $F(\mathbf{1 0}, 1 / 2), \bar{f}(\overline{\mathbf{5}},-3 / 2)$, $l^{c}(\mathbf{1}, 5 / 2), h(\mathbf{5},-1)$. The hidden sector fields transform under $S O(10)_{h} \times S U(4)_{h}$ as follows: $T(\mathbf{1 0}, 1), D(1, \mathbf{6}), \widetilde{F}(1, \mathbf{4})$. The $\widetilde{F}_{i}, \widetilde{\bar{F}}_{j}$ fields carry $\pm 1 / 2$ electric charges.

$$
\begin{aligned}
\text { Observable Sector : } & F_{0}\left(-\frac{1}{2}, 0,0,-\frac{1}{2}, 0\right) \quad F_{1}\left(-\frac{1}{2}, 0,0, \frac{1}{2}, 0\right) \\
& F_{2}\left(0,-\frac{1}{2}, 0,0,0\right) \quad \bar{f}_{2}\left(0,-\frac{1}{2}, 0,0,0\right) \quad l_{2}^{c}\left(0,-\frac{1}{2}, 0,0,0\right) \\
& F_{3}\left(0,0, \frac{1}{2}, 0,-\frac{1}{2}\right) \quad \bar{f}_{3}\left(0,0, \frac{1}{2}, 0, \frac{1}{2}\right) \quad l_{3}^{c}\left(0,0, \frac{1}{2}, 0, \frac{1}{2}\right) \\
& F_{4}\left(-\frac{1}{2}, 0,0,0,0\right) \quad \bar{F}_{4}\left(\frac{1}{2}, 0,0,0,0\right) \\
& \bar{F}_{5}\left(0, \frac{1}{2}, 0,0,0\right) \quad \bar{f}_{5}\left(0,-\frac{1}{2}, 0,0,0\right) \quad l_{5}^{c}\left(0,-\frac{1}{2}, 0,0,0\right) \\
& h_{1}(1,0,0,0,0) \quad \bar{h}_{1}(-1,0,0,0,0) \\
& h_{2}(0,1,0,0,0) \quad \bar{h}_{2}(0,-1,0,0,0) \\
& h_{3}(0,0,1,0,0) \quad \bar{h}_{3}(0,0,-1,0,0) \\
& h_{45}\left(-\frac{1}{2},-\frac{1}{2}, 0,0,0\right) \quad \bar{h}_{45}\left(\frac{1}{2}, \frac{1}{2}, 0,0,0\right)
\end{aligned}
$$

Singlets : $\quad \Phi_{12}(-1,1,0,0,0) \quad \bar{\Phi}_{12}(1,-1,0,0,0)$

$$
\begin{array}{ll}
\Phi_{23}(0,-1,1,0,0) & \bar{\Phi}_{23}(0,1,-1,0,0) \\
\Phi_{31}(1,0,-1,0,0) & \bar{\Phi}_{31}(-1,0,1,0,0) \\
\phi_{45}\left(\frac{1}{2}, \frac{1}{2}, 1,0,0\right) & \bar{\phi}_{45}\left(-\frac{1}{2},-\frac{1}{2},-1,0,0\right) \\
\phi^{+}\left(\frac{1}{2},-\frac{1}{2}, 0,0,1\right) & \bar{\phi}^{+}\left(-\frac{1}{2}, \frac{1}{2}, 0,0,-1\right) \\
\phi^{-}\left(\frac{1}{2},-\frac{1}{2}, 0,0,-1\right) & \bar{\phi}^{-}\left(-\frac{1}{2}, \frac{1}{2}, 0,0,1\right) \\
\phi_{3,4}\left(\frac{1}{2},-\frac{1}{2}, 0,0,0\right) & \bar{\phi}_{3,4}\left(-\frac{1}{2}, \frac{1}{2}, 0,0,0\right) \\
\eta_{1,2}(0,0,0,1,0) \quad \bar{\eta}_{1,2}(0,0,0,-1,0) \quad \Phi_{0,1,3,5}(0,0,0,0,0)
\end{array}
$$

Hidden Sector:

$$
\begin{array}{lll}
T_{1}\left(-\frac{1}{2}, 0, \frac{1}{2}, 0,0\right) & T_{2}\left(-\frac{1}{2},-\frac{1}{2}, 0,0,-\frac{1}{2}\right) & T_{3}\left(-\frac{1}{2}, 0, \frac{1}{2}, 0,0\right) \\
D_{1}\left(0,-\frac{1}{2}, \frac{1}{2}, \frac{1}{2}, 0\right) & D_{2}\left(0,-\frac{1}{2}, \frac{1}{2},-\frac{1}{2}, 0\right) & D_{3}\left(-\frac{1}{2}, 0, \frac{1}{2}, 0,0\right) \\
D_{4}\left(-\frac{1}{2},-\frac{1}{2}, 0,0, \frac{1}{2}\right) & D_{5}\left(0,-\frac{1}{2}, \frac{1}{2}, 0,0\right) & D_{6}\left(0, \frac{1}{2},-\frac{1}{2}, 0,0\right) \quad D_{7}\left(\frac{1}{2}, 0,-\frac{1}{2}, 0,0\right) \\
\widetilde{F}_{1}^{+}\left(-\frac{1}{4}, \frac{1}{4},-\frac{1}{4}, 0,-\frac{1}{2}\right) & \widetilde{F}_{2}^{-}\left(\frac{1}{4}, \frac{1}{4},-\frac{1}{4}, 0, \frac{1}{2}\right) & \widetilde{F}_{3}^{+}\left(\frac{1}{4},-\frac{1}{4},-\frac{1}{4}, 0, \frac{1}{2}\right) \\
\widetilde{F}_{4}^{+}\left(-\frac{1}{4}, \frac{3}{4}, \frac{1}{4}, 0,0\right) & \widetilde{F}_{5}^{+}\left(-\frac{1}{4}, \frac{1}{4},-\frac{1}{4}, 0, \frac{1}{2}\right) & \widetilde{F}_{6}^{+}\left(-\frac{1}{4}, \frac{1}{4},-\frac{1}{4}, 0,-\frac{1}{2}\right) \\
\widetilde{\bar{F}}_{1}^{-}\left(-\frac{1}{4}, \frac{1}{4}, \frac{1}{4}, \frac{1}{2},-\frac{1}{2}\right) & \widetilde{\bar{F}}_{2}^{-}\left(-\frac{1}{4}, \frac{1}{4}, \frac{1}{4},-\frac{1}{2},-\frac{1}{2}\right) & \widetilde{\bar{F}}_{3}^{-}\left(\frac{1}{4},-\frac{1}{4}, \frac{1}{4}, 0,-\frac{1}{2}\right) \\
\widetilde{\bar{F}}_{4}^{-}\left(-\frac{1}{4}, \frac{1}{4}, \frac{1}{4}, 0,-\frac{1}{2}\right) & \widetilde{\bar{F}}_{5}^{+}\left(-\frac{1}{4},-\frac{1}{4}, \frac{1}{4}, 0,-\frac{1}{2}\right) & \widetilde{\bar{F}}_{6}^{-}\left(-\frac{3}{4}, \frac{1}{4},-\frac{1}{4}, 0,0\right)
\end{array}
$$

